\title{
The Operator Product Expansion of the Lowest Higher Spin Current at Finite $N$
}

\author{
Changhyun Ahn \\ Department of Physics, Kyungpook National University, Taegu 702-701, Korea \\ ahn@knu.ac.kr
}

\begin{abstract}
For the $\mathcal{N}=2$ Kazama-Suzuki(KS) model on $\mathbf{C P}^{3}$, the lowest higher spin current with spins $\left(2, \frac{5}{2}, \frac{5}{2}, 3\right)$ is obtained from the generalized GKO coset construction. By computing the operator product expansion of this current and itself, the next higher spin current with spins $\left(3, \frac{7}{2}, \frac{7}{2}, 4\right)$ is also derived. This is a realization of the $\mathcal{N}=2 \mathcal{W}_{N+1}$ algebra with $N=3$ in the supersymmetric WZW model. By incorporating the self-coupling constant of lowest higher spin current which is known for the general $(N, k)$, we present the complete nonlinear operator product expansion of the lowest higher spin current with spins $\left(2, \frac{5}{2}, \frac{5}{2}, 3\right)$ in the $\mathcal{N}=2 \mathrm{KS}$ model on $\mathbf{C P}^{N}$ space. This should coincide with the asymptotic symmetry of the higher spin $A d S_{3}$ supergravity at the quantum level. The large $(N, k)$ 't Hooft limit and the corresponding classical nonlinear algebra are also discussed.
\end{abstract}




\section{Introduction}

There are three approaches in the study of extended symmetries in conformal field theory [1]. Initiated by Zamolodchikov [2], the approach 1 is to propose the number of extra currents with given spins and close the algebra. The direct construction of extended conformal algebra has been used with the help of computer power. The associativity for the algebra should be checked. On the other hand, developed by Fateev and Lukyanov [3, 4, 5], the approach 2 is to use the Drinfeld-Sokolov reduction based on the classical Lie algebras(or Lie superalgebras). At the quantum level, the construction of the corresponding algebra is based on the quantization of Miura transformation. The currents of the algebra in Miura basis are not primary nor quasiprimary in general. Furthermore, the approach 3, found by [6, 7], is to study the extended algebra on the basis of the Casimir construction for level 1 WZW models for simply laced Lie algebras. The difficult step is to identify the complete set of independent generating currents. By construction, the associativity is satisfied automatically.

The duality between the $W_{N}$ minimal model conformal field theories and the higher spin theory of Vasiliev on the $A d S_{3}$ has been proposed by Gaberdiel and Gopakumar in [8, 9, 10], who claim that the $W_{N}$ minimal model conformal field theory is dual, in the 't Hooft $\frac{1}{N}$ expansion, to the higher spin theory coupled to one complex scalar. The $\mathcal{N}=2$ supersymmetric extension of this duality, the higher spin $A d S_{3}$ supergravity, has been studied in [11, 12, 13 ] where the dual conformal field theory is given by $\mathcal{N}=2 \mathbf{C P}^{N}$ Kazama-Suzuki(KS) model [14, 15] in two dimensions.

Recently, for $N=4$ in the KS-model on $\mathbf{C P}^{N}$ space, the two higher spin currents with spins $\left(2, \frac{5}{2}, \frac{5}{2}, 3\right)$ and spins $\left(3, \frac{7}{2}, \frac{7}{2}, 4\right)$ in terms of constrained WZW currents are constructed and the operator product expansion of the lowest higher spin current and itself in $\mathcal{N}=2 \mathcal{W}_{N+1}$ algebra, at the linear level, is presented in [16]. By taking the large $(N, k)$ limit on the various operator product expansions in components, at the linear order, it leads to the corresponding operator product expansions in $\mathcal{N}=2$ classical algebra describing the asymptotic symmetries of bulk theory found in [13].

In this paper, we reconsider the KS coset model and find out the first nontrivial operator product expansion including the nonlinear terms. Although the two higher spin currents were found in [16], it is rather complicated to describe the operator product expansions for the various component fields explicitly. Therefore, we consider the $N=3$ case, which is the simplest one, in detail. For $\mathcal{N}=2 \mathcal{W}_{N+1}$ algebra, there exist $N$-multiplets whose first component spins are 1 (corresponding to the stress energy tensor), $2, \cdots, N$. The complete algebra for the higher spin currents consists of $\frac{1}{2} N(N-1)$ operator product expansions. 
Among them, we only compute the simplest operator product expansion between the lowest higher spin current and itself. In components, this is equivalent to $16(=4 \times 4)$ operator product expansions. It turns out that this operator product expansion looks similar to the one in $\mathcal{N}=2 \mathcal{W}_{3}$ algebra in the sense that the field contents are the same except the higher spin current with spins $\left(3, \frac{7}{2}, \frac{7}{2}, 4\right)$ and its descendant fields are absent. Of course, the coefficient functions, i.e., 1) the central charge and 2) the self-coupling constant of the lowest higher spin current, appearing in the right hand side of operator product expansion are replaced with their $N$-generalizations respectively.

In section 2 , based on the construction of higher spin current with spins $\left(2, \frac{5}{2}, \frac{5}{2}, 3\right)$ in the $\mathbf{C P}^{3} \mathrm{KS}$ coset model, we describe the complete nonlinear operator product expansion of the lowest higher spin current with spins $\left(2, \frac{5}{2}, \frac{5}{2}, 3\right)$ in the $\mathcal{N}=2 \mathrm{KS}$ model on $\mathbf{C P}^{N}$ coset space. The next higher spin current $\left(3, \frac{7}{2}, \frac{7}{2}, 4\right)$ occurs also in the right hand side of above operator product expansion.

In section 3 , we take the large $(N, k)$ limit of the operator product expansion we have found in section 2 .

In section 4, we compare the algebra we have described in section 2 with the corresponding classical algebra describing the asymptotic symmetries in the bulk theory.

In section 5, we summarize what we have found in this paper and comment on some future directions.

In the Appendices $A$ - $D$, we present some details which are necessary to sections 2,3 , and 4.

There are some partial relevant works [17]-32] which deal with the higher spin symmetry in different contexts. In [17], the three-dimensional Chern-Simons vector models are discussed. The more general coset models with larger symmetry than $W_{N}$ symmetry are described in [18]. In [19], the higher spin holographic duality is discussed in various dimensions. The states of black hole in the CFT are compared to those in the bulk in [20]. In [21], the $\mathcal{N}=2$ asymptotic symmetry is described. The nonsupersymmetric holographic dual with different group is described in [22]. In [23], the further stduy on $W_{N}$ minimal model is considered. The three-point correlation function with arbitrary spin is proposed in [24]. In [25], the light primaries in the $W_{N}$ minimal model are described at finite $N$. The CFT computation for the three-point function with spin-4 current is described in [26]. In [27], further property of correlation functions is given. The structure constants of all classical $W_{N}$ algebra are explained in [28]. In [29], the minimal model holography with even spin is proposed. For fixed 't Hooft coupling constant, the three-point function with general spin is proposed in [30]. In [31], the more precise duality property is checked by computing the partition function. The duality in 
the minimal model holography with orthogonal group is described in [32].

In particular, there are some overlaps with the recent work by Candu and Gaberdiel [33].

\section{The $\mathcal{N}=2$ quantum $\mathcal{W}_{N+1}$ algebra: the operator prod- uct expansion of lowest higher spin current}

\subsection{Review}

Let us consider the hermitian symmetric space

$$
\mathbf{C P}^{N}=\frac{S U(N+1)}{S U(N) \times U(1)}
$$

where the structure constant of the coset is inherited from the complex structure of the numerator and denominator groups. Let $G=S U(N+1)$ be an even-dimensional Lie group and $H=S U(N) \times U(1)$ be an even-dimensional subgroup. The $N$ should be even number. We introduce a complex basis for the Lie algebra in which the complex structure is diagonal. The structure constants in this basis are determined by the commutation relations of the matrix generators of Appendix $A$. It is convenient to decompose the group $G$-indices into the subgroup $H$-indices and the coset $\frac{G}{H}$-indices explicitly. The lower case middle roman indices $m, n, p, \cdots$, running from 1 to $\frac{N^{2}}{2}$, refer to the Lie algebra of $H$ while the lower case top roman indices $a, b, c, \cdots$, running from $\frac{N^{2}}{2}+1$ to $\frac{1}{2}\left[(N+1)^{2}-1\right]$, refer to the remaining Lie algebra generators corresponding to the coset $\frac{G}{H}$. We shall denote by $\bar{m}, \bar{n}, \bar{p}, \cdots$ and $\bar{a}, \bar{b}, \bar{c}, \cdots$ the complex conjugate indices. For odd $N$, we will describe the corresponding coset model in the subsection 2.2.

The ten operator product expansions between the $\mathcal{N}=2 S U(N+1)$ WZW currents are given in [16] where both the subgroup index structure and the remaining index structure are manifest.

Then the stress tensor $T(Z)$ for the supersymmetric coset model based on $\mathcal{N}=2 \mathbf{C P}^{N}$ model is obtained from [34]: quadratic and linear terms 1

$$
T(Z)=-\frac{1}{(k+N+1)}\left[J^{a} J^{\bar{a}}-f_{\bar{m} \bar{a}}^{\bar{a}} D K^{\bar{m}}-f_{m \bar{a}} \bar{a} \bar{D} K^{m}\right](Z),
$$

where $J^{a}(Z), J^{\bar{a}}(Z)$ live in the coset $\frac{G}{H}$ and $K^{m}(Z), K^{\bar{m}}(Z)$ live in the subgroup $H$. The positive integer $k$ is the level of WZW currents. One obtains the standard operator product

\footnotetext{
${ }^{1}$ The $Z$ stands for $\mathcal{N}=2$ superspace coordinates, one real bosonic coordinate $z$, and pair of two conjugate Grassman coordinates $\theta, \bar{\theta}: Z=(z, \theta, \bar{\theta})$. The complex spinor covariant derivatives are $D=\frac{\partial}{\partial \theta}-\frac{1}{2} \bar{\theta} \frac{\partial}{\partial z}$, and $\bar{D}=\frac{\partial}{\partial \bar{\theta}}-\frac{1}{2} \theta \frac{\partial}{\partial z}$.
} 
expansion of $\mathcal{N}=2$ superconformal algebra,

$$
T\left(Z_{1}\right) T\left(Z_{2}\right)=\frac{1}{z_{12}^{2}} \frac{c}{3}+\frac{\theta_{12} \bar{\theta}_{12}}{z_{12}^{2}} T\left(Z_{2}\right)-\frac{\theta_{12}}{z_{12}} D T\left(Z_{2}\right)+\frac{\bar{\theta}_{12}}{z_{12}} \bar{D} T\left(Z_{2}\right)+\frac{\theta_{12} \bar{\theta}_{12}}{z_{12}} \partial T\left(Z_{2}\right),
$$

where the central charge is

$$
c=\frac{3 N k}{N+k+1}
$$

The component result of $(2.3)$ is given by the Appendix $B$. The superfield $T(Z)$ has no $U(1)$ charge because there is no $\frac{1}{z_{12}}$-term in (2.3) . The coefficient of $\frac{\theta_{12} \bar{\theta}_{12}}{z_{12}^{2}}$ implies that the superspin of $T(Z)$ is equal to 1 or its component spins are given by $\left(1, \frac{3}{2}, \frac{3}{2}, 2\right)$.

\subsection{The $\mathrm{CP}^{3}$ coset model}

Before we are going to discuss the $N=3$ case, let us remind the $N=2$ case where the $\mathcal{N}=2 \mathcal{W}_{3}$ algebra [35, 36] is realized. The field contents are the stress tensor $T(Z)(2.2)$ and one higher spin current $W(Z)$ with spins $\left(2, \frac{5}{2}, \frac{5}{2}, 3\right)$. They can be realized by the constrained WZW currents [37] based on the approach 3. In $\mathcal{N}=2$ superspace, the only nontrivial nonlinear operator product expansion of this algebra is characterized by the operator product expansion between $W\left(Z_{1}\right)$ and $W\left(Z_{2}\right)$. On the other hand, the coefficient functions on the right hand side are completely fixed by the Jacobi identities between these two currents $T(Z)$ and $W(Z)$ along the line of the approach 1 explained in the introduction. The two approaches give the same result.

We will consider the $N=3$ case, see how the algebra looks different(compared to the $N=2$ case), and present the nonlinear algebra for general $N$ in next subsection. Let us focus on the $N=3$ case where the $S U(4)$ generators in the complex basis are given by the Appendix (A.1). The field contents of $\mathcal{N}=2 \mathcal{W}_{4}$ algebra are given by the stress tensor with spins $\left(1, \frac{3}{2}, \frac{3}{2}, 2\right)$ and two primary higher spin currents with spins $\left(2, \frac{5}{2}, \frac{5}{2}, 3\right)$, and $\left(3, \frac{7}{2}, \frac{7}{2}, 4\right)$ [38, 39] (based on the approach 1). Let us write them explicitly as follows 2 :

$$
\begin{aligned}
T(Z) & =T+\left.\theta D T\right|_{\theta=\bar{\theta}=0}+\left.\bar{\theta} \bar{D} T\right|_{\theta=\bar{\theta}=0}+\left.\theta \bar{\theta}(-1) \frac{1}{2}[D, \bar{D}] T\right|_{\theta=\bar{\theta}=0}, \\
W(Z) & =W+\left.\theta D W\right|_{\theta=\bar{\theta}=0}+\left.\bar{\theta} \bar{D} W\right|_{\theta=\bar{\theta}=0}+\left.\theta \bar{\theta}(-1) \frac{1}{2}[D, \bar{D}] W\right|_{\theta=\bar{\theta}=0}, \\
V(Z) & =V+\left.\theta D V\right|_{\theta=\bar{\theta}=0}+\left.\bar{\theta} \bar{D} V\right|_{\theta=\bar{\theta}=0}+\left.\theta \bar{\theta}(-1) \frac{1}{2}[D, \bar{D}] V\right|_{\theta=\bar{\theta}=0} .
\end{aligned}
$$

\footnotetext{
${ }^{2} \mathrm{As}$ in [16, for the component fields, instead of introducing the different notations for the various fields, we stick to use the covariant spinor derivatives to describe the three components for given one superfield. As we specify the bosonic coordinate $z$ on them, one can tell them from the corresponding superfield.
} 
The field $V(Z)$ satisfies the defining equation for the primary field condition. The coset can be described as $\mathbf{C P}^{3}$ by introducing the extra $U(1)$ 's in both the numerator and the denominator in order to have even-dimensional groups $G$ and $H$ as in (2.1)

$$
\mathbf{C P}^{3}=\frac{S U(4) \times U(1)}{S U(3) \times U(1) \times U(1)} .
$$

In the Appendix $A$, we present the generators for each group factors. The corresponding $\mathcal{N}=2$ current algebra with $S U(4)$ group in the complex basis can be obtained by inserting the structure constants and the metric. Then it is an immediate task to find the higher spin currents in terms of the fundamental currents defined in the supersymmetric WZW model(in the context of the approach 3). The stress tensor is given by (2.2). It is nontrivial to find the extra higher spin currents in the Casimir construction that includes the higher spin generators. There exist 16 independent fundamental WZW currents, $K^{m}(Z), K^{\bar{m}}(Z)$ and $J^{a}(Z), J^{\bar{a}}(Z)$ where $m, \bar{m}=1,2,3,4,5$ and $a, \bar{a}=6,7,8$. These 16 supercurrents are in fact constrained, e.g. the components $D K^{m}(z)$ and $-\frac{1}{2}[D, \bar{D}] K^{m}(z)$ of the fundamental WZW supercurrent $K^{m}(Z)$ are determined by the other 2 components $K^{m}(z)$ and $\bar{D} K^{m}(z)$. The higher spin currents in the Casimir construction must be constructed out of the independent fundamental currents.

One way to write down the lowest higher spin current $W(Z)$ with spins $\left(2, \frac{5}{2}, \frac{5}{2}, 3\right)$ is to take into account of all the possible terms for given superspin 2 in the WZW currents. Finally, it turns out, from the equation of (2.24) in [16, that the correct higher spin current with spins $\left(2, \frac{5}{2}, \frac{5}{2}, 3\right)$, takes the form 3

$$
\begin{aligned}
W(Z) & =\frac{A(k)}{\left(-8+7 k+3 k^{2}\right)}\left[\left(-8+7 k+3 k^{2}\right) f_{\bar{c} a}^{\bar{m}} f_{c \bar{b}}{ }^{n} J^{a} J^{\bar{b}} K^{m} K^{\bar{n}}\right. \\
& -2(4+k)(-1+2 k) f_{\bar{c} a}^{n} f_{c \bar{b}}{ }^{\bar{m}} J^{a} J^{\bar{b}} K^{m} K^{\bar{n}} \\
& +\frac{1}{2}\left(-56+6 k+9 k^{2}+k^{3}\right) J^{a} J^{b} J^{\bar{a}} J^{\bar{b}}+\frac{1}{2}(4+k)\left(-16+21 k+7 k^{2}\right) f_{\bar{m} a}^{b} J^{a} J^{\bar{b}} D K^{\bar{m}} \\
& -\frac{1}{2} k\left(28+11 k+k^{2}\right)\left(f_{a \bar{m}}{ }^{b} J^{a} K^{\bar{m}} D J^{\bar{b}}-f_{m a}^{b} K^{m} \bar{D} J^{a} J^{\bar{b}}\right) \\
& +\frac{1}{2}\left(-64+68 k+49 k^{2}+7 k^{3}\right) f_{m a}^{b} J^{a} J^{\bar{b}} \bar{D} K^{m}+\frac{1}{6} k(4+k)(7+k) f_{m n}{ }^{p} \bar{D} K^{m} K^{n} K^{\bar{p}} \\
& -k(4+k)^{2}(7+k) \bar{D} J^{a} D J^{\bar{a}}+2(4+k)^{2}(-1+2 k) \bar{D} K^{m} D K^{\bar{m}} \\
& +\frac{1}{4}\left(-256-112 k+48 k^{2}+35 k^{3}+5 k^{4}\right)\left(J^{a} \partial J^{\bar{a}}-\partial J^{a} J^{\bar{a}}\right) \\
& -(4+k)^{2}(-1+2 k) K^{m} \partial K^{\bar{m}}-\frac{1}{12}\left(192+160 k+24 k^{2}+3 k^{3}+k^{4}\right) \partial K^{m} K^{\bar{m}} \\
& -\frac{1}{4} k(4+k)^{2}(7+k)\left(J^{a}[D, \bar{D}] J^{\bar{a}}+[D, \bar{D}] J^{a} J^{\bar{a}}\right)
\end{aligned}
$$

\footnotetext{
${ }^{3}$ All the composite fields are normal ordered from right to left, along the line of [6, 7].
} 


$$
\begin{aligned}
& +(4+k)^{2}(-1+2 k) K^{m}[D, \bar{D}] K^{\bar{m}}-\frac{1}{12}(4+k)^{2}\left(12-17 k+k^{2}\right)[D, \bar{D}] K^{m} K^{\bar{m}} \\
& +2(4+k)^{2}(-1+2 k) D K^{m} \bar{D} K^{\bar{m}}+\frac{1}{6}(4+k)^{2}\left(6-5 k+k^{2}\right) f_{m \bar{n}} \overline{\bar{n}} \partial \bar{D} K^{m} \\
& +(4+k)^{2}(-1+2 k) f_{\bar{m} \bar{n}}^{\bar{n}} \partial D K^{\bar{m}}-\frac{1}{3}(-8+k)(4+k)^{2} f_{\bar{m} \bar{a}}^{\bar{a}} f_{n \bar{b}} \bar{b} D K^{\bar{m}} \bar{D} K^{n} \\
& +(-3+k)(4+k)^{2}\left(f_{\bar{m} \bar{b}}^{\bar{b}} J^{a} J^{\bar{a}} D K^{\bar{m}}+f_{m \bar{b}}^{\bar{b}} J^{a} J^{\bar{a}} \bar{D} K^{m}\right) \\
& +\frac{1}{6}(4+k)^{2}(7+k)\left(f_{\bar{m} \bar{a}} \overline{\bar{a}} f_{\bar{n} \bar{b}} \bar{b} D K^{\bar{m}} D K^{\bar{n}}+f_{m \bar{a}}{ }_{n \bar{b}}^{\bar{b}} \bar{D} K^{m} \bar{D} K^{n}\right) \\
& \left.+\frac{1}{2} k(4+k)^{2}(7+k) f_{\bar{m} a}^{a} \partial D K^{\bar{m}}\right](Z),
\end{aligned}
$$

where the overall constant is fixed as

$$
\begin{aligned}
A(k)^{2} & =\frac{3\left(-8+7 k+3 k^{2}\right)^{2}}{4(-1+k)(2+k)(4+k)^{6}(7+k)(-1+2 k)} \\
& =-\frac{(-9+c)^{6}\left(-54+33 c+c^{2}\right)^{2}}{1088391168(-21+c)(-1+c)(9+c)(-9+5 c)} .
\end{aligned}
$$

We will see how we obtain this result in a moment. The central charge, from (2.4), is

$$
c_{N=3}=\frac{9 k}{k+4} \text {. }
$$

The superfield $W(Z)$ consists of quartic, cubic, quadratic and linear terms. One realizes that the factor $(4+k)$ in (2.7) reflects the more general $N$-dependent factor $(N+k+1)$. We have seen the factor $(3+k)$ in the $\mathbf{C P}^{2}$ coset model and the factor $(5+k)$ in the $\mathbf{C P}^{4}$ coset model [16]. If one considers the higher spin currents with spins $\left(2, \frac{5}{2}, \frac{5}{2}, 3\right)$ for the $\mathbf{C P}^{N}$ coset model, then the factor $(N+k+1)$ should arise in various places for the composite fields.

The two conditions we use above, in order to determine the various undetermined coefficients in (2.24) of [16], are the regularity condition

$$
K^{m}\left(Z_{1}\right) W\left(Z_{2}\right)=0, \quad K^{\bar{m}}\left(Z_{1}\right) W\left(Z_{2}\right)=0,
$$

and the primary field condition

$$
T\left(Z_{1}\right) W\left(Z_{2}\right)=\frac{\theta_{12} \bar{\theta}_{12}}{z_{12}^{2}} 2 W\left(Z_{2}\right)-\frac{\theta_{12}}{z_{12}} D W\left(Z_{2}\right)+\frac{\bar{\theta}_{12}}{z_{12}} \bar{D} W\left(Z_{2}\right)+\frac{\theta_{12} \bar{\theta}_{12}}{z_{12}} \partial W\left(Z_{2}\right) .
$$

The component results for (2.11) are given by the Appendix $B$. Notice that $W(Z)$ is uncharged. All the $c$-dependent(or $k$-dependent) coefficient functions are completely fixed by these two conditions except the overall constant $A(k)$.

Now we are ready to compute the operator product expansion $W\left(Z_{1}\right) W\left(Z_{2}\right)$ from the realization of (2.7). We will use the component approach by starting with the operator product expansion between the lower spin components of $W(Z)$. 


\subsubsection{The operator product expansion $W(z) W(w)$}

Let us compute the spin-2 spin-2 operator product expansion $W\left(Z_{1}\right) W\left(Z_{2}\right)$ explicitly. As explained in [16], due to the limitation of $\mathcal{N}=2$ package [40], one resorts to the original package [41]. From the components (2.5), first of all, one can start with the operator product expansion $W(z) W(w)$ : the first component of $W\left(Z_{1}\right)$ and the first component of $W\left(Z_{2}\right)$. The first component of $W(Z)$ is obtained by taking $\theta, \bar{\theta}$ in (2.7) to zero.

- $\frac{1}{(z-w)^{4}}$-term

Let us consider the operator product expansion of $W(z) W(w)$ for the highest singular term $\frac{1}{(z-w)^{4}}$. It turns out, by taking the explicit operator product expansion between these two fields, that one obtains $\frac{6(-1+k) k(2+k)(4+k)^{5}(7+k)(-1+2 k)}{\left(-8+7 k+3 k^{2}\right)^{2}} A(k)^{2}$. Normalizing it to be $\frac{c}{2}$ where the central charge $c$ is given by (2.9), one sees the normalization factor (2.8).

- $\frac{1}{(z-w)^{3}}$-term

Let us move on the next singular term $\frac{1}{(z-w)^{3}}$. Such a term is not allowed by the symmetry of the operator product expansion $W(z) W(w)$. Suppose that there is such a singular term. After reversing the arguments $z$ and $w$ and expanding around $w$, the same term will appear with an opposite sign. This implies that it is identically zero.

- $\frac{1}{(z-w)^{2}}$-term

The next singular term $\frac{1}{(z-w)^{2}}$ contains many different combinations of spin-2 fields in terms of WZW currents. The possible spin-2 fields(some of them are primary and others are not primary) as $W(w),[D, \bar{D}] T(w), T^{2}(w)$ and $\partial T(w)$. However, the last one is not allowed because it is a descendant of $T(w)$ and the latter did not appear in the more singular terms. Then it is straightforward to rewrite this singular term in terms of above three spin-2 fields by introducing three undetermined coefficients which depend on the level $k$. The easiest way to determine the relative coefficients is to compute the singular term using the representation (2.7) and equate the result to the ansatz above. Then the unknown three coefficients are completely fixed. In particular, the coefficient of $W(w)$ turns out to be $\frac{4(-3+k)(1+k)(4+k)^{3}}{-8+7 k+3 k^{2}} A(k)$ by explicit computations. In our notation, this is equal to $2 \alpha$ and with (2.8), one gets the self-coupling constant, the coefficient function appearing in the $W(w)$ term of the right hand side in the $W(z) W(w)$,

$$
\alpha_{N=3}^{2}=\frac{3(-3+k)^{2}(1+k)^{2}}{(-1+k)(2+k)(7+k)(-1+2 k)}=-\frac{(27-7 c)^{2}(3+c)^{2}}{2(-21+c)(-1+c)(9+c)(-9+5 c)}
$$

where the level $k$ is replaced by the central charge $c(2.9)$ in the last expression. We will see that this self-coupling constant is consistent with the general expression with arbitrary $N$ that can be obtained from the Jacobi identity [33]. 
- $\frac{1}{(z-w)}$-term

Let us describe the final lowest singular term $\frac{1}{(z-w)}$. The possible spin-3 terms are characterized by

$$
\begin{aligned}
& T^{3}(w), T W(w), V(w),[D, \bar{D}] W(w), T[D, \bar{D}] T(w), \\
& \bar{D} T D T(w), \partial^{2} T(w), \partial W(w), \partial[D, \bar{D}] T(w), \text { and } \partial T T(w) .
\end{aligned}
$$

For the first six independent terms, due to the symmetry described in the $\frac{1}{(z-w)^{3}}$ term, we do not see them in this singular term, and we do not see the $\partial^{2} T(w)$-term because there is no $T(w)$-term in the right hand side as above. Then, we are left with the last three independent terms. They are exactly the corresponding descendant terms for the spin-2 fields $W(w)$, $[D, \bar{D}] T(w)$, and $T T(w)$. It turns out, by explicit computations, that all the coefficients are given by the half of the coefficients given in the singular term $\frac{1}{(z-w)^{2}}$. Also note that $\partial T^{2}(w)=2 \partial T T(w)=2 T \partial T(w)$ with the operator product expansion $T(z) T(w)$ in the Appendix $B$. The relative coefficient $\frac{1}{2}$ behavior is a consequence of conformal invariance [42, 1].

Therefore, the operator product expansion $W(z) W(w)$ is summarized in (C.1). Basically, this is the same as the one in $N=2$ case [16]. Of course, the central charge and the selfcoupling constant in this case are different and given by (2.9) and (2.12).

\subsubsection{The operator product expansion $W(z) \bar{D} W(w)$}

Now let us consider the other operator product expansion. In order to compute the spin2 spin- $\frac{5}{2}$ operator product expansion $W(z) \bar{D} W(w)$, one should know the explicit form for the primary spin- $\frac{5}{2}$ field $\bar{D} W(w)$. This can be read off from the operator product expansion $\bar{D} T(z) W(w)$ given in $(B .2)$. By looking at the singular term $\frac{1}{(z-w)}$, it turns out the spin- $\frac{5}{2}$ field $\bar{D} W(w)$ consists of 189 WZW currents which will not be written down here.

For given $\bar{D} W(w)$ (or one can obtain this in closed form by acting $\bar{D}$ on the field $W(Z)$ (2.7) and putting $\theta, \bar{\theta}$ to zero), by explicit computation of $W(z) \bar{D} W(w)$, one can easily identify the highest singular term $\frac{1}{(z-w)^{3}}$ with $-3 D T(w)$. The second order singular term can contain all spin- $\frac{5}{2}$ fields, $\bar{D} W(w), \partial \bar{D} T(w), T \bar{D} T(w)$ and its conjugate fields. Because $\bar{D} W$ has charge -1 , only the first three spin- $\frac{5}{2}$ fields appear on the right hand side. As before, we can write down an ansatz for this singular term in terms of these spin- $\frac{5}{2}$ fields and solve it by comparing with the singular term computed with the GKO approach. The final first-order singular term can be written in terms of following spin- $\frac{7}{2}$ fields:

$$
\begin{aligned}
& \partial \bar{D} W(w), \partial^{2} \bar{D} T(w), T \bar{D} W(w), T T \bar{D} T(w), \bar{D} T W(w), \\
& \bar{D} T[D, \bar{D}] T(w), \partial \bar{D} T T(w), \partial T \bar{D} T(w) \text { and } \bar{D} V(w) .
\end{aligned}
$$


Compared to the $N=2$ case, there exists an extra term $\bar{D} V(w)$ which is the third component of superfield $V\left(Z_{2}\right)$ with spins $\left(3, \frac{7}{2}, \frac{7}{2}, 4\right)(2.5)$. We will come to this issue after the discussion of next operator product expansion where one realizes the presence of $V(w)$, the first component of $V\left(Z_{2}\right)$. Therefore, the remaining nonzero terms after subtracting the eight terms with correct coefficient functions in this singular term are really the third component of primary superfield $V\left(Z_{2}\right)$. The final result is summarized by $(C .2)$.

\subsubsection{The operator product expansion $W(z) D W(w)$}

Similarly, one can analyze the spin- 2 spin- $\frac{5}{2}$ operator product expansion $W(z) D W(w)$. The explicit form for $D W(w)$ can be obtained from the operator product expansion $D T(z) W(w)$ in $(B .2)$ or by acting $D$ on the $W(Z)$ and putting $\theta, \bar{\theta}$ to zero. Since we know the form for $D W(w)$ explicitly, one can compute the operator product expansion. In this case, the spin- $\frac{7}{2}$ field $D V(w)$ arises at the lowest singular term as well as the conjugated fields of (2.14). This is also presented in (C.2) with explicit coefficient functions.

\subsubsection{The operator product expansion $W(z)(-1) \frac{1}{2}[D, \bar{D}] W(w)$}

Now let us find out the explicit form for the spin-3 field $-\frac{1}{2}[D, \bar{D}] W(z)$ in order to compute the spin-2 spin-3 operator product expansion $W(z)(-1) \frac{1}{2}[D, \bar{D}] W(w)$. From the operator product expansion $D T(z) \bar{D} W(w)$ in $(B .2)$, one can read off this primary spin-3 field. The

singular term $\frac{1}{(z-w)}$ contains this spin-3 field as well as $\frac{1}{2} \partial W(w)$. Then it is easy to obtain the spin-3 field which consists of 1065 independent WZW currents(Also one can obtain this field from the superfield $W(Z)(2.7)$ with the derivatives $D$ and $\bar{D}$ ).

Let us consider the operator product expansion $W(z)(-1) \frac{1}{2}[D, \bar{D}] W(w)$. See also the Appendix (C.3). The fourth-order singular term has primary spin-1 field $T(w)$ and the coefficient is given by 3 . There is no third-order singular term. The second-order singular term contains the spin-3 fields. Among 10 possible terms we described before in (2.13) , there are no $\partial T T(w)$ and $\partial W(w)$. This is consistent with the fact that the spin-2 fields $T^{2}(w)$ and $W(w)$ do not occur in the third-order singular term. After collecting 7-independent terms with appropriate coefficient functions, we are left with a nonzero spin-3 field which is denoted by $3 V(w)$ where

$$
\begin{aligned}
V(w) & =\frac{24(-1+2 k)(11+2 k)\left(32-12 k-11 k^{2}+6 k^{3}\right)}{(-1+k)(2+k)(4+k)^{3}(-4+5 k)(8+5 k)(-16+11 k)} J^{6} J^{7} J^{8} J^{\overline{6}} J^{\overline{7}} J^{\overline{8}}(w) \\
& +\frac{9(-1+2 k)}{(-1+k)(2+k)(4+k)^{4}(7+k)}\left[K^{2} K^{3} K^{4} K^{2} K^{\overline{3}} K^{\overline{4}}-K^{1} K^{2} K^{4} K^{\overline{1}} K^{\overline{2}} K^{\overline{4}}\right](w) \\
& + \text { other } 1282 \text { terms, }
\end{aligned}
$$


where the abbreviated terms contain fifth-order, fourth-order, $\cdots$, second-order and first-order terms in constrained WZW currents. Of course, it would be interesting to write down (2.15) using the group theory structure constants as in (2.7) 4. The overall scale factor of spin-3 field can be fixed after computing the operator product expansion $V(z) V(w)$ which will not be done in this paper. For the first-order singular term, one should consider the possible spin-4 fields. One expects that the descendant fields for the above eight spin-3 fields(i.e., the derivative terms) appear. Moreover, the two fields $\bar{D} T D W(w)$ and $D T \bar{D} W(w)$ also occur.

Since the spin-3 field $V(w)$ is found, one can compute the operator product expansion $D T(z) V(w)$ and the first-order singular term should be equal to $-D V(w)$ from the primary field condition in (B.2). One checks that the first-order singular term coincides with the remaining terms described in the first-order singular term of the operator product expansion $W(z) D W(w)$ as above. This confirms that we have the correct normalization of components relative to each other. Similarly, the operator product expansion $\bar{D} T(z) V(w)$ provides the correct expression for $\bar{D} V(w)$.

\subsubsection{The operator product expansion $D W(z) \bar{D} W(w)$}

What about the spin-4 field $(-1) \frac{1}{2}[D, \bar{D}] V(z)$ ? One can compute the covariant derivatives of a superfield only if one knows all of its components. Or it can be determined as in the previous analysis for $(-1) \frac{1}{2}[D, \bar{D}] W(z)$. From the operator product expansion $D T(z) \bar{D} V(w)$ in $(B .2)$, one can read off this primary spin-4 field. The singular term $\frac{1}{(z-w)}$ contains this spin-4 field as well as $\frac{1}{2} \partial V(w)$. This spin-4 field occurs in the operator product expansion $D W(z) \bar{D} W(w)$. For example, the second component of $W\left(Z_{1}\right)$ and the third component of $W\left(Z_{2}\right)$. The relevant singular term is given by the first-order singular term. One can exhaust all the possible uncharged spin-4 fields as follows:

$$
\begin{aligned}
& \partial^{2} W(w), \partial^{2}[D, \bar{D}] T(w), T[D, \bar{D}] W(w), T T[D, \bar{D}] T(w), T \bar{D} T D T(w), \\
& \bar{D} T D W(w), D T \bar{D} W(w), \partial \bar{D} T D T(w), \partial D T \bar{D} T(w),[D, \bar{D}] T W(w), \\
& {[D, \bar{D}] T[D, \bar{D}] T(w), \partial T \partial T(w), \partial^{2} T T(w),[D, \bar{D}] V(w), \partial[D, \bar{D}] W(w),} \\
& \partial^{3} T(w), \partial T W(w), T \partial W(w), \partial[D, \bar{D}] T T(w), \partial T[D, \bar{D}] T(w), \\
& \partial T T T(w), \partial V(w), T^{4}(w), T T W(w), T V(w), W^{2}(w) .
\end{aligned}
$$

\footnotetext{
${ }^{4}$ From the expressions (2.2), and (2.7), one can write down the above spin-3 fields (2.13) except $V(w)$ itself in terms of the structure constants and WZW currents. Now we introduce the arbitrary coefficients inside of each spin-3 field. Then one can check whether the known spin-3 field (2.15) can be written in terms of the combinations of spin-3 fields in (2.13) or not. In other words, are the undetermined coefficients uniquely fixed? Eventhough there will be extra terms, it will not be so difficult to write them using the summation index structure.
} 
Among these 26-terms (2.16), it turns out that the coefficients for the last five terms are vanishing 5 . It is rather complicated to fix all the coefficients explicitly using the GKO representation and make sure that the remaining terms after subtracting everybody besides $[D, \bar{D}] V$ can be interpreted as the $\mathbf{C P}^{4}$ coset field of spin-4. In other words, this new field is primary field of spin 4 under the stress tensor. Moreover, the fact that this is coset field implies that one should have the regularity conditions $K^{m}(z)[D, \bar{D}] V(w)=0, \bar{D} K^{m}(z)[D, \bar{D}] V(w)=0$, $K^{\bar{m}}(z)[D, \bar{D}] V(w)=0$, and $D K^{\bar{m}}(z)[D, \bar{D}] V(w)=0$. We have checked these identities explicitly.

So far, we have checked the following operator product expansions,

$$
W(z) W(w), W(z) D W(w), W(z) \bar{D} W(w), W(z)(-1) \frac{1}{2}[D, \bar{D}] W(w), D W(z) \bar{D} W(w) \cdot(2.17)
$$

One can proceed further with the remaining operator product expansions. However, it is rather complicated procedure to check all the nontrivial operator product expansions. Instead, by resorting to $\mathcal{N}=2$ supersymmetry, one can reexpress without any ambiguity the above operator product expansions (2.17) using $\mathcal{N}=2$ superspace formalism. Now one can write one single operator product expansion $W\left(Z_{1}\right) W\left(Z_{2}\right)$ explicitly which will be presented in next subsection. Then the unchecked operator product expansions can be read off from this and they will appear in the Appendix $C$ completely.

In summary, one concludes, compared to the $N=2$ case [37, 43], that the operator product expansion of the lowest higher spin current with spins $\left(2, \frac{5}{2}, \frac{5}{2}, 3\right)$ in $\mathcal{N}=2 \mathrm{KS}$ coset model on $\mathbf{C P}^{3}(2.6)$ has the next higher spin current with spins $\left(3, \frac{7}{2}, \frac{7}{2}, 4\right)(2.15)$. One can rewrite all the structure constants in terms of $c$ and the self-coupling constant (2.12). All dependence on $N$ is contained in these two parameters, provided the higher spin field $\left(3, \frac{7}{2}, \frac{7}{2}, 4\right)$ is normalized as in equation (2.18).

\subsection{The $\mathrm{CP}^{N}$ coset model}

So far, we have considered the $N=2$ case in [37], the $N=3$ case in this paper, and the $N=4$ case in [16]. As we increase the $N(>4)$, we expect to have the similar features.

1) The self-coupling constant, that appears in the $W(w)$-dependent terms, changes according to $N$ but its expression is known for general $N$ [38],

\footnotetext{
5 In the package [41, sometimes it takes too much time to compute the first-order pole terms by using simply the command "OPESimplify[OPEPole[1][ope], Factor]". Instead, one defines result $\equiv$ OPEPole[1][ope]; and $L \equiv$ GetOperators[result]; and then computes "Sum[Simplify[Coefficients[result, $L[[i]]]] *$ $L[[i]],\{i, 1$, Length $[L]\}]$ ". In other words, one gets the raw expression by "result", gets the independent terms by $L$, simplifies the coefficient functions appearing in the list of $L$, and then sums over the product between the independent fields and the coefficient functions. This leads to the final fist-order pole terms with simplified coefficient functions.
} 
2) the higher spin currents, whose normalization should be fixed, occur and

3) the $c$-dependent coefficient functions in terms of $c$, that appear in $W(w)$-independent and -dependent terms in the right hand side, do not change. Of course, the central charge itself depends on $N$.

For example, for $N=4$ the self-coupling constant is known and one could take the same $c$-dependent coefficient functions as in the $N=3$ case.

What about the presence of an extra higher spin current? If we denote the higher spin current $X(Z)$ with spins $\left(4, \frac{9}{2}, \frac{9}{2}, 5\right)$ which is one of the field contents in $\mathcal{N}=2 \mathcal{W}_{5}$ algebra, by dimensional analysis, the spin- 4 field $X\left(Z_{2}\right)$ can appear in the singular term $\frac{\theta_{12} \bar{\theta}_{12}}{z_{12}}$ but it seems that this is not the case. Actually, this is direct consequence of the Jacobi identities [33. Eventually, this higher spin current will appear in other operator product expansions between the higher spin currents whose spins are greater than the spin of lowest higher spin current.

The final operator product expansion of the superspin 2 current and itself which is the main result of this paper, from the component results of (2.17), can be written as

$$
\begin{aligned}
& W\left(Z_{1}\right) W\left(Z_{2}\right)=\frac{1}{z_{12}^{4}} \frac{c}{2}+\frac{\theta_{12} \bar{\theta}_{12}}{z_{12}^{4}} 3 T\left(Z_{2}\right)+\frac{\bar{\theta}_{12}}{z_{12}^{3}} 3 \bar{D} T\left(Z_{2}\right)-\frac{\theta_{12}}{z_{12}^{3}} 3 D T\left(Z_{2}\right)+\frac{\theta_{12} \bar{\theta}_{12}}{z_{12}^{3}} 3 \partial T\left(Z_{2}\right) \\
& +\frac{1}{z_{12}^{2}}\left[2 \alpha W-\frac{c}{(-1+c)}[D, \bar{D}] T-\frac{3}{(-1+c)} T^{2}\right]\left(Z_{2}\right) \\
& +\frac{\bar{\theta}_{12}}{z_{12}^{2}}\left[\alpha \bar{D} W+\frac{(-3+2 c)}{(-1+c)} \partial \bar{D} T-\frac{3}{(-1+c)} T \bar{D} T\right]\left(Z_{2}\right) \\
& +\frac{\theta_{12}}{z_{12}^{2}}\left[\alpha D W-\frac{(-3+2 c)}{(-1+c)} \partial D T-\frac{3}{(-1+c)} T D T\right]\left(Z_{2}\right) \\
& +\frac{\theta_{12} \bar{\theta}_{12}}{z_{12}^{2}}\left[-\frac{3(-8+c)}{2(-12+5 c)} \alpha[D, \bar{D}] W-\frac{9 c(-12+5 c)}{4(-1+c)(6+c)(-3+2 c)} \partial[D, \bar{D}] T\right. \\
& +\frac{3\left(18-15 c+2 c^{2}+2 c^{3}\right)}{2(-1+c)(6+c)(-3+2 c)} \partial^{2} T+3 V+\frac{42}{(-12+5 c)} \alpha T W \\
& -\frac{3\left(36-9 c+8 c^{2}\right)}{2(-1+c)(6+c)(-3+2 c)} T[D, \bar{D}] T-\frac{9(3+4 c)}{(-1+c)(6+c)(-3+2 c)} T^{3} \\
& \left.-\frac{9 c(-12+5 c)}{(-1+c)(6+c)(-3+2 c)} \bar{D} T D T\right]\left(Z_{2}\right) \\
& +\frac{1}{z_{12}}\left[\alpha \partial W-\frac{c}{2(-1+c)} \partial[D, \bar{D}] T-\frac{3}{(-1+c)} \partial T T\right]\left(Z_{2}\right) \\
& +\frac{\bar{\theta}_{12}}{z_{12}}\left[\frac{3(-6+c)(-1+c)}{(3+c)(-12+5 c)} \alpha \partial \bar{D} W+\frac{3 c\left(9+3 c+2 c^{2}\right)}{4(-1+c)(6+c)(-3+2 c)} \partial^{2} \bar{D} T+\bar{D} V\right. \\
& -\frac{6(-15+c)}{(3+c)(-12+5 c)} \alpha T \bar{D} W-\frac{9(3+4 c)}{(-1+c)(6+c)(-3+2 c)} T T \bar{D} T
\end{aligned}
$$




$$
\begin{aligned}
& +\frac{54(-1+c)}{(3+c)(-12+5 c)} \alpha \bar{D} T W-\frac{27 c}{2(6+c)(-3+2 c)} \bar{D} T[D, \bar{D}] T \\
& \left.-\frac{3\left(-18+24 c+c^{2}\right)}{(-1+c)(6+c)(-3+2 c)} \partial \bar{D} T T-\frac{9(-6+c)}{2(6+c)(-3+2 c)} \partial T \bar{D} T\right]\left(Z_{2}\right) \\
& +\frac{\theta_{12}}{z_{12}}\left[\frac{3(-6+c)(-1+c)}{(3+c)(-12+5 c)} \alpha \partial D W-\frac{3 c\left(9-3 c+c^{2}\right)}{2(-1+c)(6+c)(-3+2 c)} \partial^{2} D T-D V\right. \\
& +\frac{6(-15+c)}{(3+c)(-12+5 c)} \alpha T D W+\frac{9(3+4 c)}{(-1+c)(6+c)(-3+2 c)} T T D T \\
& +\frac{27 c}{2(6+c)(-3+2 c)}[D, \bar{D}] T D T-\frac{54(-1+c)}{(3+c)(-12+5 c)} \alpha D T W \\
& \left.-\frac{3\left(-18+24 c+c^{2}\right)}{(-1+c)(6+c)(-3+2 c)} \partial D T T-\frac{9(-6+c)}{2(6+c)(-3+2 c)} \partial T D T\right]\left(Z_{2}\right) \\
& +\frac{\theta_{12} \bar{\theta}_{12}}{z_{12}}\left[-\frac{(-15+c) c}{(3+c)(-12+5 c)} \alpha \partial[D, \bar{D}] W+\frac{\left(-18-3 c-2 c^{2}+2 c^{3}\right)}{2(-1+c)(6+c)(-3+2 c)} \partial^{3} T+2 \partial V\right. \\
& +\frac{18(6+c)}{(3+c)(-12+5 c)} \alpha T \partial W+\frac{12(3+4 c)}{(3+c)(-12+5 c)} \alpha \partial T W \\
& +\frac{6}{(3+c)} \alpha \bar{D} T D W-\frac{6 c(-12+5 c)}{(-1+c)(6+c)(-3+2 c)} \partial \bar{D} T D T \\
& -\frac{6\left(9-3 c+c^{2}\right)}{(-1+c)(6+c)(-3+2 c)} \partial[D, \bar{D}] T T+\frac{6}{(3+c)} \alpha D T \bar{D} W \\
& +\frac{6 c(-12+5 c)}{(-1+c)(6+c)(-3+2 c)} \partial D T \bar{D} T-\frac{3 c(3+4 c)}{(-1+c)(6+c)(-3+2 c)} \partial T[D, \bar{D}] T \\
& \left.-\frac{18(3+4 c)}{(-1+c)(6+c)(-3+2 c)} \partial T T T\right]\left(Z_{2}\right)+\cdots
\end{aligned}
$$

Here 6 the central charge and self-coupling constant are given by

$$
\begin{aligned}
c & =\frac{3 N k}{N+k+1}, \\
\alpha^{2} & =\frac{3(1+k)^{2}(k-N)^{2}(1+N)^{2}}{(-1+k)(-1+N)(1+2 k+N)(1+k+2 N)(-1-N+k(-1+3 N))} \\
& =\frac{(3+c)^{2}\left(c+2 c N-3 N^{2}\right)^{2}}{(-1+c)(3-c+6 N)(-1+N)(c+3 N)(-3 N+c(2+N))} .
\end{aligned}
$$

The $N$ - and $k$-dependences in the right hand side occur through these two values. The full quantum operator product expansion is characterized by two free parameters, the central

\footnotetext{
${ }^{6}$ The coupling constant $c_{22,3}$ in front of $V\left(Z_{2}\right)$ in (2.18) was found in 33. From the equations (2.18), (2.19), (2.20) and (3.30) in [33], one can write down the square of this coupling constant as $c_{22,3}^{2}=\frac{3(3+c)(-12+5 c)}{(6+c)(-3+2 c)}+$ $\frac{6(-15+c)(-1+c) \alpha^{2}}{(3+c)(-12+5 c)}$ with (2.20). This implies that the correct normalization for this field should be $\hat{V}\left(Z_{2}\right)$ with $c_{22,3} \hat{V}\left(Z_{2}\right)=3 V\left(Z_{2}\right)$. We thank the referee for pointing out this.
} 
charge and the self-coupling constant. For $N=2$ case, one easily sees that the above operator product expansion leads to the previous results in [35] by putting the spin-3 field $V(w)$ (and its descendant fields) and spin- $\frac{5}{2}$ fields $D V(w)$ and $\bar{D} V(w)$ to vanish. For fixed $N$, the central charge and the self-coupling constant depend on only the level $k$. For $N=3$ case, as we explained before, the general expression for (2.18) reduces to the findings in previous subsection. One also sees that $\alpha$ becomes the one in $(\underline{2.12})$ when $N=3$. In the $\frac{\theta_{12} \bar{\theta}_{12}}{z_{12}}$-singular term, we do not see the possible terms with spins $\left(4, \frac{9}{2}, \frac{9}{2}, 5\right)$ like as $T^{4}\left(Z_{2}\right), \operatorname{TTW}\left(Z_{2}\right), W^{2}\left(Z_{2}\right)$ or $T V\left(Z_{2}\right) 7$.

Note that the 29 nonlinear terms in (2.18) which appear in the last entries of each singular term are the new we observe and the remaining 26 linear terms are found in [16]. We present the 16 component operator product expansions in the Appendix $C$.

In order to compare the bulk theory computations, one should also consider the higher spin currents in non-primary basis. By subtracting(or excising) the spin-1 current, one can construct the following spin-2 current [35] by adding the quadratic piece in $T(z)$ :

$$
-\frac{1}{2}[D \widetilde{\bar{D}}] T(z)=-\frac{1}{2}[D, \bar{D}] T(z)-\frac{3}{2 c} T^{2}(z)
$$

The corresponding central charge is given by $(c-1)$ and the operator product expansion satisfies

$$
\begin{aligned}
(-1) \frac{1}{2}[D \widetilde{\bar{D}}] T(z)(-1) \frac{1}{2}[D \widetilde{\bar{D}}] T(w) & =\frac{1}{(z-w)^{4}} \frac{(-1+c)}{2}+\frac{1}{(z-w)^{2}} 2(-1) \frac{1}{2}[D \widetilde{\bar{D}}] T(w) \\
& +\frac{1}{(z-w)}(-1) \frac{1}{2} \partial[D \widetilde{\bar{D}}] T(w)+\cdots,
\end{aligned}
$$

corresponding to $(3.21 a)$ of [13]. Then there are no any singular terms with spin-1 current

$$
(-1) \frac{1}{2}[\overline{D, \bar{D}}] T(z) T(w)=0 .
$$

This corresponds to (3.21b) of [13]. This comes from the coefficient $\frac{3}{2 c}$ in the second term of (2.21). Furthermore, the previous spin- $\frac{3}{2}$ currents in primary basis are not primary fields, due to the extra term in (2.21), but the corresponding operator product expansions contain the nonlinear terms

$$
(-1) \frac{1}{2}[D \widetilde{\bar{D}}] T(z)(D T \pm \bar{D} T)(w)=\frac{1}{(z-w)^{2}}\left[\frac{3}{2}-\frac{3}{2 c}\right][D T \pm \bar{D} T](w)
$$

\footnotetext{
${ }^{7}$ We have noticed this fact in previous section in the context of component results. These terms appear in the different singular term of the operator product expansion of $W\left(Z_{1}\right) T W\left(Z_{2}\right)$. This implies that one should modify the fourth component of the higher spin current with spins $\left(2, \frac{5}{2}, \frac{5}{2}, 3\right)$ by adding the first component of the higher spin current $T W(Z)$ with spins $\left(3, \frac{7}{2}, \frac{7}{2}, 4\right)$. We will see this feature in detail soon. Moreover, the possible 10 terms, among (2.16), with same spins, coming from $T\left(Z_{2}\right)$ and $W\left(Z_{2}\right)$ with the appropriate derivatives, are not present in the term $\frac{\theta_{12} \bar{\theta}_{12}}{z_{12}}$ of (2.18).
} 


$$
+\frac{1}{(z-w)}\left[\partial(D T \pm \bar{D} T)-\frac{3}{c} T(D T \mp \bar{D} T)\right](w)+\cdots
$$

Even in the $c \rightarrow \infty$ limit, the nonlinear terms $\frac{3}{c} T(D T \mp \bar{D} T)$ arise while $c$-dependent linear term vanishes [44, 45] where the only $c$-independent factor can survive in the linear field term and the $\frac{1}{c}$-dependent factor can survive in the quadratic field term. Strictly speaking, the currents $D T(w)$ and $\bar{D} T(w)$ are not primary fields of spin $\frac{3}{2}$ under the stress energy tensor (2.21) even at the linear order for the finite $c$. They become primary fields $c \rightarrow \infty$ limit at the linear order. One realizes that the corresponding bulk expressions are given by (3.21d) and $(3.21 e)$ of [13]. One also checks the other relations from the operator product expansions (B.1). In particular, as soon as the spin-2 term $[D, \bar{D}] T(w)$ in the right hand side of the operator product expansion appears, one should rewrite it using the relation (2.21). This implies that the operator product expansions in the spin- $\frac{3}{2}$ and spin- $\frac{3}{2}$ contain the nonlinear term $T^{2}(w)$ as in $(3.21 h)$ and $(3.21 j)$ of [13] 8. Therefore, the four component fields $T(z)$, $D T(z), \bar{D} T(z)$, and $-\frac{1}{2}[D, \overline{\bar{D}}] T(z)$ of $\mathcal{N}=2$ superconformal algebra, with an appropriate classical limit, coincide with those quantities in the bulk theory [13]. Their operator product expansions with stress energy tensor are given by (2.22), (2.23), and (2.24).

What about the higher spin currents? Due to this modification of stress energy tensor, one should also add the extra term in the spin-3 current, in order to preserve the primary field condition, as follows:

$$
-\frac{1}{2}[D, \overline{\bar{D}}] W(z)=-\frac{1}{2}[D, \bar{D}] W(z)-\frac{6}{c} T W(z) .
$$

One can easily check the standard primary field condition with spin 3,

$$
\begin{aligned}
(-1) \frac{1}{2}[D, \widetilde{\bar{D}}] T(z)(-1) \frac{1}{2}[D, \widetilde{\bar{D}}] W(w) & =\frac{1}{(z-w)^{2}} 3(-1) \frac{1}{2}[D, \widetilde{\bar{D}}] W(w) \\
& +\frac{1}{(z-w)}(-1) \frac{1}{2} \partial[D, \widetilde{\bar{D}}] W(w)+\cdots
\end{aligned}
$$

corresponding to $(3.26 a)$ of 13 . For the spin-2 current $W(w)$, the primary field condition does not change because the extra term $T^{2}(z)$ in (2.21) does not produce any extra singular structure, i.e., $T(z) W(w)=0$,

$$
(-1) \frac{1}{2}[D \widetilde{\bar{D}}] T(z) W(w)=\frac{1}{(z-w)^{2}} 2 W(w)+\frac{1}{(z-w)} \partial W(w)+\cdots .
$$

\footnotetext{
${ }^{8}$ For the $(3.21 c)$ of [13], one has $T(z) T(w)$ of the Appendix $C$. For the $(3.21 f)$ and $(3.21 g)$ of [13], one has $T(z)(D T \mp \bar{D} T)(w)$ which gives $(D T \pm \bar{D} T)(w)$ in the right hand side of the operator product expansion. For $(3.21 i)$ of [13], one has $(D T-\bar{D} T)(z)(D T+\bar{D} T)(w)$ of the Appendix $C$.
} 
For the spin- $\frac{5}{2}$ currents, one sees the similar behavior as in (2.24)

$$
\begin{aligned}
& (-1) \frac{1}{2}[D, \widetilde{\bar{D}}] T(z)(D W \pm \bar{D} W)(w)=\frac{1}{(z-w)^{2}}\left[\frac{5}{2}-\frac{3}{2 c}\right][D W \pm \bar{D} W](w) \\
& +\frac{1}{(z-w)}\left[\partial(D W \pm \bar{D} W)-\frac{3}{c} T(D W \mp \bar{D} W)\right](w)+\cdots
\end{aligned}
$$

In this case, the nonlinear terms are $\frac{3}{c} T(D W \mp \bar{D} W)$ which do not vanish in the $c \rightarrow \infty$ limit. This corresponds to the equation (3.26b) of [13]. As before, these spin- $\frac{5}{2}$ fields are not primary field. One can easily see that there is no singular term in the operator product expansion between $T(z)$ and the field (2.25) which corresponds to (3.26c) of [13]. For the operator product expansions between $(D T \pm \bar{D} T)(z)$ with the current (2.25) in the Appendix $B$, one has the nonlinear terms from the equations $((3.26 f)$ and $(3.26 j))$. Similarly, from the operator product expansions between $(D W \pm \bar{D} W)$ and $(D T \pm \bar{D} T)$ in the Appendix $B$, the nonlinear terms in the right hand side are the same as the ones $((3.26 h)$ and $(3.26 m))$ in $[13$. 9. Therefore, the four component fields $W(z), D W(z), \bar{D} W(z)$, and $-\frac{1}{2}[D, \widetilde{\bar{D}}] W(z)$ of $\mathcal{N}=2$ lowest higher spin current coincide with those quantities in the bulk theory [13] after the $c \rightarrow \infty$. Their operator product expansions with stress energy tensor are given by (2.26), (2.27), and (2.28).

For the comparison with the classical asymptotic symmetry algebra in the bulk theory, one should consider the modified two quantities (2.21) and (2.25). In other words, the old quantities for the spin-2 and spin-3 currents have the extra terms. Then one expects that the composite operators containing the fields $T^{2}(z)$ and $T W(z)$ and the various derivative terms(coming from the normal ordering procedure) occur in the right hand side. In $\mathcal{N}=2$ superspace, one should also consider the operator product expansions $T\left(Z_{1}\right) T W\left(Z_{2}\right), T^{2}\left(Z_{1}\right) T W\left(Z_{2}\right)$, $T^{2}\left(Z_{1}\right) W\left(Z_{2}\right)$, and $W\left(Z_{1}\right) T W\left(Z_{2}\right)$. We will come to this issue in next sections.

\section{The large $(N, k)$ 't Hooft limit of $\mathcal{N}=2$ quantum $\mathcal{W}_{N+1}$ algebra}

The large $(N, k)$ limit with fixed 't Hooft coupling constant is characterized by 8 ]

$$
c(N, k)=\frac{3 N k}{N+k+1} \longrightarrow 3(1-\lambda) N, \quad \lambda \equiv \frac{N}{N+k} .
$$

\footnotetext{
${ }^{9}$ For the linear terms, the relations $(3.26 d)$ and $(3.26 e)$ in 13 correspond to the operator product expansions $T(z)(D W \pm \bar{D} W)(w)$ in the Appendix B. The equations $(3.26 g)$ and $(3.26 i)$ correspond to the operator product expansions $(D T-\bar{D} T)(z) W(w)$ and $(D T-\bar{D} T)(z)(D W+\bar{D} W)(w)$. Furthermore, the equations $(3.26 k)$ and $(3.26 l)$ correspond to the operator product expansions $(D T+\bar{D} T)(z) W(w)$ and $(D T+\bar{D} T)(z)(D W-\bar{D} W)(w)$ in the Appendix $B$.
} 
For this limit, the self coupling constant behaves as follows [16]:

$$
\alpha(N, k)^{2} \longrightarrow-\frac{(-1+2 \lambda)^{2}}{(-2+\lambda)(1+\lambda)} .
$$

One can think of other limit 9] where for fixed $N$, one takes the large $c$ limit. Then, from the finite $(N, c)$ expression for the self-coupling constant (2.20), one obtains $\alpha(N, k)^{2} \rightarrow$ $-\frac{(2 N+1)^{2}}{(N-1)(N+2)}$.

Then it is easy to see that under the large $(N, k)$ limit the operator product expansion (2.18) becomes the following classical limit

$$
\begin{aligned}
& W\left(Z_{1}\right) W\left(Z_{2}\right)=\frac{1}{z_{12}^{4}} \frac{c}{2}+\frac{\theta_{12} \bar{\theta}_{12}}{z_{12}^{4}} 3 T\left(Z_{2}\right)+\frac{\bar{\theta}_{12}}{z_{12}^{3}} 3 \bar{D} T\left(Z_{2}\right) \\
& -\frac{\theta_{12}}{z_{12}^{3}} 3 D T\left(Z_{2}\right)+\frac{\theta_{12} \bar{\theta}_{12}}{z_{12}^{3}} 3 \partial T\left(Z_{2}\right)+\frac{1}{z_{12}^{2}}\left[2 \alpha W-[D, \bar{D}] T-\frac{3}{c} T^{2}\right]\left(Z_{2}\right) \\
& +\frac{\bar{\theta}_{12}}{z_{12}^{2}}\left[\alpha \bar{D} W+2 \partial \bar{D} T-\frac{3}{c} T \bar{D} T\right]\left(Z_{2}\right)+\frac{\theta_{12}}{z_{12}^{2}}\left[\alpha D W-2 \partial D T-\frac{3}{c} T D T\right]\left(Z_{2}\right) \\
& +\frac{\theta_{12} \bar{\theta}_{12}}{z_{12}^{2}}\left[-\frac{3}{10} \alpha[D, \bar{D}] W+\frac{3}{2} \partial^{2} T+3 V+\frac{42}{5 c} \alpha T W-\frac{6}{c} T[D, \bar{D}] T\right. \\
& \left.-\frac{18}{c^{2}} T^{3}-\frac{45}{2 c} \bar{D} T D T\right]\left(Z_{2}\right)+\frac{1}{z_{12}}\left[\alpha \partial W-\frac{1}{2} \partial[D, \bar{D}] T-\frac{3}{c} \partial T T\right]\left(Z_{2}\right) \\
& +\frac{\bar{\theta}_{12}}{z_{12}}\left[\frac{3}{5} \alpha \partial \bar{D} W+\frac{3}{4} \partial^{2} \bar{D} T+\bar{D} V-\frac{6}{5 c} \alpha T \bar{D} W-\frac{18}{c^{2}} T T \bar{D} T+\frac{54}{5 c} \alpha \bar{D} T W\right. \\
& \left.-\frac{27}{4 c} \bar{D} T[D, \bar{D}] T-\frac{3}{2 c} \partial \bar{D} T T-\frac{9}{4 c} \partial T \bar{D} T\right]\left(Z_{2}\right) \\
& +\frac{\theta_{12}}{z_{12}}\left[\frac{3}{5} \alpha \partial D W-\frac{3}{4} \partial^{2} D T-D V+\frac{6}{5 c} \alpha T D W+\frac{18}{c^{2}} T T D T\right. \\
& \left.+\frac{27}{4 c}[D, \bar{D}] T D T-\frac{54}{5 c} \alpha D T W-\frac{3}{2 c} \partial D T T-\frac{9}{4 c} \partial T D T\right]\left(Z_{2}\right) \\
& +\frac{\theta_{12} \bar{\theta}_{12}}{z_{12}}\left[-\frac{1}{5} \alpha \partial[D, \bar{D}] W+\frac{1}{2} \partial^{3} T+2 \partial V+\frac{18}{5 c} \alpha T \partial W+\frac{48}{5 c} \alpha \partial T W\right. \\
& +\frac{6}{c} \alpha \bar{D} T D W-\frac{15}{c} \partial \bar{D} T D T-\frac{3}{c} \partial[D, \bar{D}] T T+\frac{6}{c} \alpha D T \bar{D} W \\
& \left.+\frac{15}{c} \partial D T \bar{D} T-\frac{6}{c} \partial T[D, \bar{D}] T-\frac{36}{c^{2}} \partial T T T\right]\left(Z_{2}\right)+\cdots
\end{aligned}
$$

The term $\partial[D, \bar{D}] T(w)$ in the $\frac{\theta_{12} \bar{\theta}_{12}}{z_{12}^{2}}$ term goes away [44, 45, 46]. See also the previous work [45] for the reduction of classical algebra from the quantum algebra where the precise limiting procedure is given. It is known that there exists an $\mathcal{N}=2$ supersymmetric version of classical $W_{3}$ algebra in $\mathcal{N}=2$ superspace [47] corresponding to the component approach [48].

One sees the relative coefficients appearing in $\frac{\theta_{12}}{z_{12}}$ term, $\frac{27}{4 c}, \frac{3}{2 c}$, and $\frac{9}{4 c}$ are consistent with those in [47] where the corresponding coefficients are given by $\frac{36}{c_{I K}}, \frac{8}{c_{I K}}$, and $\frac{12}{c_{I K}}$ in spin- $\frac{7}{2}$ field. 
One can find that the relative coefficients $\frac{6}{5 c}$ and $\frac{54}{5 c}$ correspond to the values $\frac{8}{c_{I K}}$ and $\frac{72}{c_{I K}}$. We do not have to worry about the ordering between the fields in the classical expression. For example, $\operatorname{DTW}\left(Z_{2}\right)$ in the above singular term is the same as $W D T\left(Z_{2}\right)$. One can interchange any fields in the classical algebra (3.3). For the fermionic fields, there exists minus sign between the interchanging of any two fermionic fields. For example, $\bar{D} T D T\left(Z_{2}\right)=-D T \bar{D} T\left(Z_{2}\right)$. In the package [41, this can be done by using "SetOPEOptions[OPEMethod, ClassicalOPEs]". Moreover, the relative coefficients appearing in $\frac{\theta_{12} \bar{\theta}_{12}}{z_{12}^{2}}$ term, $\frac{6}{c}$, and $\frac{45}{2 c}$ correspond to the coefficients $\frac{32}{c_{I K}}$, and $\frac{120}{c_{I K}}$ in spin-3 field.

Of course, the component approach results are read off from the Appendix $C$ with an appropriate limit for the central charge. One can easily check that the above result (3.3) contains the operator product expansion [47] with an appropriate normalization.

\section{The $\mathcal{N}=2$ classical $\mathcal{W}_{\infty}^{\mathrm{cl}}[\lambda]$ algebra in the bulk theory}

In this section, we would like to see the operator product expansions appearing in the asymptotic symmetry of the higher spin $A d S_{3}$ supergravity theory from our findings in section 2 . In the Appendix $C$, we have our component operator product expansions completely. As we observed, the spin-3 field is modified via (2.25). Then from primary basis to nonprimary basis, one should recompute the operator product expansions containing this spin-3 field. Furthermore, one should change the old fields appearing in the right hand side into the new fields, with the defining equations (2.21) and (2.25).

Let us consider the three operator product expansions containing the spin-2 current. Other remaining operator product expansions will be given in the Appendix $D$.

In this section, we take the large $(N, k)$ 't Hooft limit with (3.1) and (3.2) on the algebra we have found in previous section or in the Appendix $C$.

\subsection{The operator product expansion $W(z) W(w)$}

For example, the spin-2 and spin-2 operator product expansion can be written as

$$
\begin{aligned}
& W(z) W(w)=\frac{1}{(z-w)^{4}} \frac{c}{2}+\frac{1}{(z-w)^{2}}\left[2 \alpha W-\frac{c}{(-1+c)}[\widetilde{D, \bar{D}}] T\right](w) \\
& +\frac{1}{(z-w)}\left[\alpha \partial W-\frac{c}{2(-1+c)} \partial[\widetilde{D \overline{\bar{D}}}] T\right](w)+\cdots \\
& \longrightarrow \frac{1}{(z-w)^{4}} \frac{c}{2}+\frac{1}{(z-w)^{2}}[2 \alpha W-[D \widetilde{\bar{D}}] T](w)
\end{aligned}
$$




$$
+\frac{1}{(z-w)}\left[\alpha \partial W-\frac{1}{2} \partial[\widetilde{D, \bar{D}}] T\right](w)+\cdots
$$

where the old stress energy tensor is replaced by the new stress energy tensor (2.21). In the classical $c \rightarrow \infty$ limit, one finds the coincidence with the equation (3.27) of [13]. Note that the central charge $c$ depends on $(N, k)$ and the self-coupling constant $\alpha$ depends on $(N, k)$ also. At the quantum level, one should use (4.1) rather than (4.2). The difference between (4.1) and (4.2) is the fact that the two parameters $c$ and $\alpha$ are replaced by their limiting values (3.1) and (3.2) respectively. The field contents do not change. In general, one expects that there exist some fields in the quantum operator product expansion which will disappear in the classical limit. Note that the relative coefficient $\frac{1}{2}$ between the second-order and first-order singular terms(i.e., the latter can be written as $\frac{1}{2}$ times the total derivative of the former) is well-known numerical factor which can be observed from the conformal invariance.

\subsection{The operator product expansion $W(z)(D W \pm \bar{D} W)(w)$}

Let us consider the spin-2 and spin- $\frac{5}{2}$ operator product expansions. Again, from the operator product expansion $(C .2)$, one obtains

$$
\begin{aligned}
& W(z)(D W \pm \bar{D} W)(w)=\frac{1}{(z-w)^{3}} 3[D T \mp \bar{D} T](w) \\
& +\frac{1}{(z-w)^{2}}\left[\alpha(D W \pm \bar{D} W)+\frac{c}{(-1+c)} \partial(D T \mp \bar{D} T)-\frac{3}{(-1+c)} T(D T \pm \bar{D} T)\right](w) \\
& +\frac{1}{(z-w)}\left[(D V \mp \bar{D} V)+\frac{2\left(-27+12 c+c^{2}\right)}{(3+c)(-12+5 c)} \alpha \partial(D W \pm \bar{D} W)\right. \\
& \mp \frac{(-3+c)(3+c)(-9+2 c)}{4(-1+c)(6+c)(-3+2 c)} \partial^{2} \bar{D} T-\frac{6(-15+c)}{(3+c)(-12+5 c)} \alpha T(D W \mp \bar{D} W) \\
& +\frac{9(-15+c)}{2(-1+c)(6+c)(-3+2 c)} T T(D T \mp \bar{D} T)+\frac{54(-1+c)}{(3+c)(-12+5 c)} \alpha(D T \mp \bar{D} T) W \\
& -\frac{27 c}{2(6+c)(-3+2 c)}([D, \bar{D}] T D T \mp \bar{D} T[D \bar{D}] T)+\frac{(-15+c)(-3+c) c}{2(-1+c)(6+c)(-3+2 c)} \partial^{2} D T \\
& -\frac{3(-15+c) c}{(-1+c)(6+c)(-3+2 c)}\left(\partial D T T+\frac{1}{2} \partial T D T\right) \\
& \left.\mp \frac{3\left(-54+39 c+c^{2}\right)}{2(-1+c)(6+c)(-3+2 c)} \partial T \bar{D} T \mp \frac{3\left(-27+12 c+c^{2}\right)}{(-1+c)(6+c)(-3+2 c)} \partial \bar{D} T T\right](w)+\cdots \\
& \longrightarrow \frac{1}{(z-w)^{3}} 3[D T \mp \bar{D} T](w) \\
& +\frac{1}{(z-w)^{2}}\left[\alpha(D W \pm \bar{D} W)+\partial(D T \mp \bar{D} T)-\frac{3}{c} T(D T \pm \bar{D} T)\right](w)
\end{aligned}
$$




$$
\begin{aligned}
& +\frac{1}{(z-w)}\left[(D V \mp \bar{D} V)+\frac{2}{5} \alpha \partial(D W \pm \bar{D} W)-\frac{6}{5 c} \alpha T(D W \mp \bar{D} W)\right. \\
& -\frac{9}{4 c^{2}} T T(D T \mp \bar{D} T)+\frac{54}{5 c} \alpha(D T \mp \bar{D} T) W-\frac{27}{4 c}[\overline{D, \bar{D}}] T(D T \mp \bar{D} T) \\
& \left.+\frac{1}{4} \partial^{2}(D T \mp \bar{D} T)-\frac{3}{2 c}(\partial D T \pm \partial \bar{D} T) T-\frac{3}{4 c} \partial T(D T \pm \bar{D} T)\right](w)+\cdots
\end{aligned}
$$

The 14 nonlinear terms in the classical expression are exactly the same as the ones in [13]. They are denoted by $B F_{\frac{5}{2}, \frac{5}{2}}$ and $B F_{\frac{5}{2}, 3}$ respectively. The ordering in the composite operators is important at the quantum level. This will give rise to the different coefficient function in the derivative terms. For example, $(D T \mp \bar{D} T) W(w), \mp \bar{D} T[\widetilde{D, \bar{D}}] T(w)$ and $(\partial D T \pm \partial \bar{D} T) T(w)$. However, in the classical limit we do not have to worry about the ordering because the different ordering gives the same result, as we described in previous section. Note that the relative coefficients $-\frac{3}{c},-\frac{3}{2 c}$, and $-\frac{3}{4 c}$ correspond to $4,2,1$ in the $a_{\frac{3}{2}} \psi_{\frac{3}{2}}\left(a_{\frac{3}{2}} \psi_{2}\right.$ in the second sign) terms [13]. One realizes that the relative coefficients $\frac{54}{5 c}$ and $-\frac{6}{5 c}\left(=-\frac{1}{9} \times \frac{54}{5 c}\right)$ in the $\alpha$-dependent terms give the correct values in [13].

The relative coefficients $\frac{1}{3}$ and $\frac{1}{12}$ appearing in the descendant fields for $(D T \mp \bar{D} T)(w)$ can be obtained the formula [42, 1], $\frac{1}{n !} \times \frac{\Gamma\left(h_{i}-h_{j}+h_{k}+n\right)}{\Gamma\left(h_{i}-h_{j}+h_{k}\right)} \times \frac{\Gamma\left(2 h_{k}\right)}{\Gamma\left(2 h_{k}+n\right)}$ where $h_{i}, h_{j}$, and $h_{k}$ are the conformal dimensions in the operator product expansion $\phi^{i}(z) \phi^{j}(w) \sim \phi^{k}(w)$ and $n$ is the number of derivatives of $\phi^{k}(w)$. Let us compute for the case of the field $(D T \pm \bar{D} T)(w)$ and its descendant field. One sees that $h_{i}=2, h_{j}=\frac{5}{2}$, and $h_{k}=\frac{3}{2}$ in the primary basis. Then the coefficient of $\partial(D T \pm \bar{D} T)(w)$ can be read off from $\frac{1}{1 !} \times \frac{\Gamma\left(2-\frac{5}{2}+\frac{3}{2}+1\right)}{\Gamma\left(2-\frac{5}{2}+\frac{3}{2}\right)} \times \frac{\Gamma\left(2 \times \frac{3}{2}\right)}{\Gamma\left(2 \times \frac{3}{2}+1\right)}=\frac{1}{3}$ where $n=1$ and the coefficient of $\partial^{2}(D T \pm \bar{D} T)(w)$ is given by $\frac{1}{2 !} \times \frac{\Gamma\left(2-\frac{5}{2}+\frac{3}{2}+2\right)}{\Gamma\left(2-\frac{5}{2}+\frac{3}{2}\right)} \times \frac{\Gamma\left(2 \times \frac{3}{2}\right)}{\Gamma\left(2 \times \frac{3}{2}+2\right)}=\frac{1}{12}$ where $n=2$. For given coefficient 3 in the $(D T \pm \bar{D} T)$, the former becomes $3 \times \frac{1}{3}=1$ and the latter becomes $3 \times \frac{1}{12}=\frac{1}{4}$ as in (4.3).

\subsection{The operator product expansion $\left.W(z)(-1) \frac{1}{2} \widetilde{[D}, \bar{D}\right] W(w)$}

In this case, one should compute the extra operator product expansion between $W(z)$ and $T W(w)$. Let us describe the operator product expansion between the spin-2 and spin-3, by adding this extra contribution to (C.3),

$$
\begin{aligned}
& W(z)(-1) \frac{1}{2}[D, \bar{D}] W(w)=\frac{1}{(z-w)^{2}}\left[-\frac{3(-8+c)}{2(-12+5 c)} \alpha[D, \widetilde{\bar{D}}] W\right. \\
& -\frac{9 c(-12+5 c)}{4(-1+c)(6+c)(-3+2 c)} \partial[D \widetilde{\bar{D}}] T+\frac{27(-12+5 c)}{(-1+c)(6+c)(-3+2 c)} \partial T T \\
& +\frac{27(-12+5 c)}{2(-1+c)(6+c)(-3+2 c)} T[D \widetilde{\bar{D}}] T-\frac{54(-12+5 c)}{(-1+c) c(6+c)(-3+2 c)} T^{3}
\end{aligned}
$$




$$
\begin{aligned}
& \left.-\frac{9 c(-12+5 c)}{(-1+c)(6+c)(-3+2 c)} \bar{D} T D T-\frac{3 c(-12+5 c)}{2(-1+c)(6+c)(-3+2 c)} \partial^{2} T+3 V\right](w) \\
& +\frac{1}{(z-w)}\left[-\frac{\left(-72+15 c+c^{2}\right)}{2(3+c)(-12+5 c)} \alpha \partial[D, \widetilde{D}] W+\frac{18}{c(3+c)} \alpha T \partial W\right. \\
& -\frac{6}{(3+c)} \alpha(\bar{D} T D W+D T \bar{D} W)-\frac{3 c(-12+5 c)}{(-1+c)(6+c)(-3+2 c)}(\partial \bar{D} T D T-\partial D T \bar{D} T) \\
& +\frac{9(-12+5 c)}{2(-1+c)(6+c)(-3+2 c)} \partial[D, \widetilde{\bar{D}}] T T+\frac{36}{c(3+c)} \alpha \partial T W \\
& +\frac{9(-12+5 c)}{2(-1+c)(6+c)(-3+2 c)} \partial T[\widetilde{\bar{D}}] T-\frac{54(-12+5 c)}{(-1+c) c(6+c)(-3+2 c)} \partial T T T \\
& \left.-\frac{(-12+5 c)}{4(-1+c)(-3+2 c)} \partial^{3} T+\partial V\right](w)+\cdots \\
& \longrightarrow \frac{1}{(z-w)^{2}}\left[-\frac{3}{10} \alpha[D, \widetilde{D}] W-\frac{45}{2 c} \bar{D} T D T+3 V\right](w) \\
& +\frac{1}{(z-w)}\left[-\frac{1}{10} \alpha \partial[D, \widetilde{\bar{D}}] W-\frac{6}{c} \alpha(\bar{D} T D W+D T \bar{D} W)-\frac{15}{2 c}(\partial \bar{D} T D T-\partial D T \bar{D} T)\right. \\
& +\partial V](w)+\cdots
\end{aligned}
$$

There is no fourth-order singular term due to the particular combination of spin-3 field in (2.25). See the equation (C.3) where the fourth-order singular term occurs. Compared to the previous two cases, many terms in the quantum operator product expansion vanish in the classical limit. In the $\alpha$-independent nonlinear term for the classical limit, the relative coefficients give the correct values and also the coefficients $-\frac{15}{2 c}$ and $\frac{15}{2 c}$ are consistent with those in the bulk theory [13. Note that there is an extra term $\bar{D} T D T(w)$ in the second singular term and this doesn't appear in the classical algebra. One can rewrite the last two nonlinear terms in the first-order singular term as $-\frac{15}{2 c} \partial(\bar{D} T D T)(w)$. Therefore, the presence

of $-\frac{45}{2 c} \bar{D} T D T(w)$ generates its above descendant field. The relative coefficient $\frac{1}{3}$ is true from the conformal invariance.

We will present the other remaining operator product expansions in the Appendix $D$ given by $(D .1),(D .2),(D .3)$, and (D.4). As in (4.4), for the operator product expansions containing the new spin-3 field, one should recombine the contributions from $T W(z)$. Furthermore, one should write down the old fields using the two relations (2.21) and (2.25).

\section{Conclusions and outlook}

We have found the complete "nonlinear" operator product expansion, characterized by (2.18) together with (2.19) and (2.20), of the lowest higher spin current with spins $\left(2, \frac{5}{2}, \frac{5}{2}, 3\right)$ in the 
$\mathcal{N}=2 \mathrm{KS}$ model on $\mathbf{C P}^{N}$ space. In component approach, they are given in $(C .1)-(C .10)$ in primary basis. The asymptotic symmetry of the higher spin $A d S_{3}$ supergravity, at the quantum level, should preserve the $\mathcal{N}=2 \mathcal{W}_{N+1}$ algebra which contains the above nonlinear operator product expansion 10 .

- It is an open problem to describe the quantum analysis of $A d S_{3}$ supergravity(or gravity in the context of [49]) bulk theory. As noticed in (2.24) and (2.28), one should take into account the quantum behavior(or normal ordered product in the composite operators). We expect that there are additional $c$-dependent terms in the variations of various generators with different spins in the asymptotic symmetry algebra.

- The field contents for the $\mathcal{N}=2 \mathcal{W}_{N+1}$ algebra are given by $T(Z), W(Z), V(Z), X(Z)$, a current with spins $\left(5, \frac{11}{2}, \frac{11}{2}, 6\right), \cdots$, a current with spins $\left(N, N+\frac{1}{2}, N+\frac{1}{2}, N+1\right)$. It is an open problem to construct these currents in terms of $\mathcal{N}=2 \mathrm{WZW}$ constrained currents. The first step is how to generalize the current (2.7), satisfying the conditions (2.10) and (2.11), to arbitrary $N$. Once this current is found, then in principle, one can proceed to compute the operator product expansion, by hand, to determine other higher spin currents.

- So far, we have considered the operator product expansion of $W\left(Z_{1}\right) W\left(Z_{2}\right)$. It would be interesting to see the remaining operator product expansions for the higher spin currents. For example, $W\left(Z_{1}\right) V\left(Z_{2}\right)$ and $V\left(Z_{1}\right) V\left(Z_{2}\right)$ for general $N$. As in previous paragraph, in order to obtain these, it is necessary to find out the $W(Z)$ for general $N$. For $\mathcal{N}=2 \mathcal{W}_{4}$ algebra where $N=3$, these are constructed in [38], along the line of the approach 1 , but the explicit results are still missing.

- As described in the introduction, the approach 2 is based on the quantum Miura transformation. Some of the currents in the Fateev-Lukyanov construction are found in [50, 51, 13]. It is an open problem to compute all the other currents systematically and see whether these satisfy the above operator product expansion (2.18). In this case, in general, the fields are not primary. Therefore, the nontrivial task is to find the right primary fields with $N$-dependent coefficient functions.

- One can also consider the bosonic subalgebra of (2.18) for particular $N$. For $N=2$, this analysis was done in [35]. For $N=3$, the subalgebra should contain the bosonic $W_{4}$ algebra. In the notation of [1], one denotes it as $\mathcal{W}(2,3,4)$. The field contents of this algebra are given by the stress tensor of spin 2, one higher spin current of spin 3, and other higher spin current of spin 4. It would be interesting to obtain the algebra found in [52, 53]. The main thing

\footnotetext{
${ }^{10}$ The nonlinear structure of the 't Hooft limit of (2.18) is in principle fixed by $\mathcal{N}=2$ conformal invariance. Therefore, these nonlinear terms are bound to match with the ones obtained from the Drinfeld-Sokolov reduction of supersymmetric higher spin algebra if the linear ones do. We thank the referee for pointing this out.
} 
is to identify the correct primary fields for given fields coming from $T(Z), W(Z)$ and $V(Z)$. Similarly, the $N=4$ case corresponds to the bosonic $W_{5}$ algebra denoted by $\mathcal{W}(2,3,4,5)$. It is an open problem to obtain the algebra in [54, 55] from our result.

\section{Acknowledgments}

CA would like to thank A. Wisskirchen for sending the paper [39] and thank the referee for pointing out many comments which are very helpful to improve the draft. This work was supported by the Mid-career Researcher Program through the National Research Foundation of Korea (NRF) grant funded by the Korean government (MEST) (No. 2012045385). CA acknowledges warm hospitality from the School of Liberal Arts (and Institute of Convergence Fundamental Studies), Seoul National University of Science and Technology.

\section{Appendix A The generators and structure constants of $U(1) \times S U(4)$ in complex basis}

Let us describe the 5 generators $T_{m}$ where $m=1,2, \cdots, 5$ and the remaining 3 generators $T_{a}$ where $a=6,7,8$ as follows:

$$
\begin{aligned}
& T_{1}=\left(\begin{array}{llll}
0 & 0 & 0 & 0 \\
1 & 0 & 0 & 0 \\
0 & 0 & 0 & 0 \\
0 & 0 & 0 & 0
\end{array}\right), T_{2}=\left(\begin{array}{llll}
0 & 0 & 0 & 0 \\
0 & 0 & 0 & 0 \\
1 & 0 & 0 & 0 \\
0 & 0 & 0 & 0
\end{array}\right), T_{3}=\left(\begin{array}{llll}
0 & 0 & 0 & 0 \\
0 & 0 & 0 & 0 \\
0 & 1 & 0 & 0 \\
0 & 0 & 0 & 0
\end{array}\right), \\
& T_{4}=\left(\begin{array}{cccc}
\frac{i}{2}+\frac{1}{\sqrt{12}} & 0 & 0 & 0 \\
0 & -\frac{i}{2}+\frac{1}{\sqrt{12}} & 0 & 0 \\
0 & 0 & -\frac{2}{\sqrt{12}} & 0 \\
0 & 0 & 0 & 0
\end{array}\right) \\
& T_{5}=\left(\begin{array}{cccc}
\frac{i}{\sqrt{24}}+\frac{\sqrt{5}}{\sqrt{40}} & 0 & 0 & 0 \\
0 & \frac{i}{\sqrt{24}}+\frac{\sqrt{5}}{\sqrt{40}} & 0 & 0 \\
0 & 0 & \frac{i}{\sqrt{24}}+\frac{\sqrt{5}}{\sqrt{40}} & 0 \\
0 & 0 & 0 & -\frac{3 i}{\sqrt{24}}+\frac{\sqrt{5}}{\sqrt{40}}
\end{array}\right) \text {, } \\
& T_{6}=\left(\begin{array}{llll}
0 & 0 & 0 & 0 \\
0 & 0 & 0 & 0 \\
0 & 0 & 0 & 0 \\
1 & 0 & 0 & 0
\end{array}\right), T_{7}=\left(\begin{array}{llll}
0 & 0 & 0 & 0 \\
0 & 0 & 0 & 0 \\
0 & 0 & 0 & 0 \\
0 & 1 & 0 & 0
\end{array}\right), T_{8}=\left(\begin{array}{llll}
0 & 0 & 0 & 0 \\
0 & 0 & 0 & 0 \\
0 & 0 & 0 & 0 \\
0 & 0 & 1 & 0
\end{array}\right) .
\end{aligned}
$$

Note that $T_{4}=i H_{1}+H_{2}$ and $T_{5}=i H_{3}+H_{4}$ where the three Cartan generators are

given $H_{1}=\frac{1}{2} \operatorname{diag}(1,-1,0,0), H_{2}=\frac{1}{\sqrt{12}} \operatorname{diag}(1,1,-2,0), H_{3}=\frac{1}{\sqrt{24}} \operatorname{diag}(1,1,1,-3)$ and $H_{4}=\frac{\sqrt{5}}{\sqrt{40}} \operatorname{diag}(1,1,1,1)$. The generators have been normalized so that $\operatorname{tr} T_{a} T_{\bar{b}}=\delta_{a b}$ and 
$\operatorname{tr} T_{m} T_{\bar{n}}=\delta_{m n}$. The conjugated generators $T_{\bar{m}}$ and $T_{\bar{a}}$ can be obtained from $T_{\bar{m}}=T_{m}^{\dagger}$ and $T_{\bar{a}}=T_{a}^{\dagger}$. One can easily see that the 8 generators $T_{m}, T_{\bar{m}}$ where $m=1,2, \cdots, 4$ consist of the $S U(3)$ subgroup generators. The remaining diagonal generators $\left(T_{5}+T_{\overline{5}}\right)$ and $\left(T_{5}-T_{\overline{5}}\right)$ correspond to the two $U(1)$ 's in the subgroup $H$ of the coset model (2.6) and the generator $\left(T_{5}+T_{5}\right)$ corresponds to the $U(1)$ in the numerator.

\section{Appendix B The operator product expansions (2.3) and (2.11) in the component approach}

Practically, it is often useful to compute the operator product expansions in component approach. Now we would like to rewrite the $\mathcal{N}=2$ superspace formula (2.3) as follows:

$$
\begin{aligned}
T(z) T(w) & =\frac{1}{(z-w)^{2}} \frac{c}{3}+\cdots \\
T(z) D T(w) & =\frac{1}{(z-w)} D T(w)+\cdots, \\
T(z) \bar{D} T(w) & =-\frac{1}{(z-w)} \bar{D} T(w)+\cdots, \\
T(z)[D, \bar{D}] T(w) & =-\frac{1}{(z-w)^{2}} 2 T(w)+\cdots, \\
D T(z) T(w) & =-\frac{1}{(z-w)} D T(w)+\cdots, \\
D T(z) \bar{D} T(w) & =\frac{1}{(z-w)^{3}} \frac{c}{3}+\frac{1}{(z-w)^{2}} T(w) \\
& +\frac{1}{(z-w)} \frac{1}{2}[-[D, \bar{D}] T+\partial T](w)+\cdots, \\
D T(z)[D, \bar{D}] T(w) & =-\frac{1}{(z-w)^{2}} 3 D T(w)-\frac{1}{(z-w)} \partial D T(w)+\cdots, \\
\bar{D} T(z) T(w) & =\frac{1}{(z-w)} \bar{D} T(w)+\cdots, \\
\bar{D} T(z) D T(w) & =\frac{1}{(z-w)^{3}} \frac{c}{3}-\frac{1}{(z-w)^{2}} T(w) \\
& -\frac{1}{(z-w)} \frac{1}{2}[[D, \bar{D}] T+\partial T](w)+\cdots, \\
\bar{D} T(z)[D, \bar{D}] T(w) & =-\frac{1}{(z-w)^{2}} 3 \bar{D} T(w)-\frac{1}{(z-w)} \partial \bar{D} T(w)+\cdots, \\
(-1) \frac{1}{2}[D, \bar{D}] T(z) T(w) & =\frac{1}{(z-w)^{2}} T(w)+\frac{1}{(z-w)} \partial T(w)+\cdots, \\
\hline D, \bar{D}] T(z) D T(w) & =\frac{1}{(z-w)^{2}} \frac{3}{2} D T(w)+\frac{1}{(z-w)} \partial D T(w)+\cdots,
\end{aligned}
$$




$$
\begin{aligned}
(-1) \frac{1}{2}[D, \bar{D}] T(z) \bar{D} T(w) & =\frac{1}{(z-w)^{2}} \frac{3}{2} \bar{D} T(w)+\frac{1}{(z-w)} \partial \bar{D} T(w)+\cdots \\
(-1) \frac{1}{2}[D, \bar{D}] T(z)[D, \bar{D}] T(w) & =-\frac{1}{(z-w)^{4}} c+\frac{1}{(z-w)^{2}} 2[D, \bar{D}] T(w) \\
& +\frac{1}{(z-w)} \partial[D, \bar{D}] T(w)+\cdots
\end{aligned}
$$

Similarly, the operator product expansions between the stress energy tensor and the higher spin current $\Phi$ with spins $\left(\Delta, \Delta+\frac{1}{2}, \Delta+\frac{1}{2}, \Delta+1\right)$ can be written as

$$
\begin{aligned}
& T(z) D \Phi(w)=\frac{1}{(z-w)} D \Phi(w)+\cdots, \\
& T(z) \bar{D} \Phi(w)=-\frac{1}{(z-w)} \bar{D} \Phi(w)+\cdots \\
& T(z)[D, \bar{D}] \Phi(w)=-\frac{1}{(z-w)^{2}} 2 \Delta \Phi(w)+\cdots, \\
& D T(z) \Phi(w)=-\frac{1}{(z-w)} D \Phi(w)+\cdots, \\
& D T(z) \bar{D} \Phi(w)=\frac{1}{(z-w)^{2}} \Delta \Phi(w)+\frac{1}{(z-w)} \frac{1}{2}[-[D, \bar{D}] \Phi+\partial \Phi](w)+\cdots, \\
& D T(z)[D, \bar{D}] \Phi(w)=-\frac{1}{(z-w)^{2}}(2 \Delta+1) D \Phi(w)-\frac{1}{(z-w)} \partial D \Phi(w)+\cdots, \\
& \bar{D} T(z) \Phi(w)=\frac{1}{(z-w)} \bar{D} \Phi(w)+\cdots, \\
& \bar{D} T(z) D \Phi(w)=-\frac{1}{(z-w)^{2}} \Delta \Phi(w)-\frac{1}{(z-w)} \frac{1}{2}[[D, \bar{D}] \Phi+\partial \Phi](w)+\cdots, \\
& \bar{D} T(z)[D, \bar{D}] \Phi(w)=-\frac{1}{(z-w)^{2}}(2 \Delta+1) \bar{D} \Phi(w)-\frac{1}{(z-w)} \partial \bar{D} \Phi(w)+\cdots, \\
& (-1) \frac{1}{2}[D, \bar{D}] T(z) \Phi(w)=\frac{1}{(z-w)^{2}} \Delta \Phi(w)+\frac{1}{(z-w)} \partial \Phi(w)+\cdots, \\
& (-1) \frac{1}{2}[D, \bar{D}] T(z) D \Phi(w)=\frac{1}{(z-w)^{2}}\left(\Delta+\frac{1}{2}\right) D \Phi(w)+\frac{1}{(z-w)} \partial D \Phi(w)+\cdots, \\
& (-1) \frac{1}{2}[D, \bar{D}] T(z) \bar{D} \Phi(w)=\frac{1}{(z-w)^{2}}\left(\Delta+\frac{1}{2}\right) \bar{D} \Phi(w)+\frac{1}{(z-w)} \partial \bar{D} \Phi(w)+\cdots, \\
& (-1) \frac{1}{2}[D, \bar{D}] T(z)[D, \bar{D}] \Phi(w)=\frac{1}{(z-w)^{2}}(\Delta+1)[D, \bar{D}] \Phi(w)+\frac{1}{(z-w)} \partial[D, \bar{D}] \Phi(w) \\
& +\cdots \text {. }
\end{aligned}
$$

All the component fields except the fourth component of $T(Z)$ in (2.5) are primary, from (B.1) and (B.2), with respect to the stress energy tensor $(-1) \frac{1}{2}[D, \bar{D}] T(z)$. As we described in section 2, under the modified stress energy tensor (2.21), some of them are not primary. In general, once we know the stress energy tensor and the first component of any primary 
field, then other three components of this primary field are determined by the primary field condition. We used this property all the time in this paper.

\section{Appendix C The operator product expansions of $\mathcal{N}=2$ quantum $\mathcal{W}_{N+1}$ algebra in component approach: Primary basis}

The operator product expansion of spin-2 and spin-2, from the relation (2.18), can be written as 11

$$
\begin{aligned}
& W(z) W(w)=\frac{1}{(z-w)^{4}} \frac{c}{2} \\
& +\frac{1}{(z-w)^{2}}\left[2 \alpha W-\frac{c}{(-1+c)}[D, \bar{D}] T-\frac{3}{(-1+c)} T^{2}\right](w) \\
& +\frac{1}{(z-w)}\left[\alpha \partial W-\frac{c}{2(-1+c)} \partial[D, \bar{D}] T-\frac{3}{(-1+c)} \partial T T\right](w)+\cdots \\
& \longrightarrow \frac{1}{(z-w)^{4}} \frac{c}{2}+\frac{1}{(z-w)^{2}}\left[2 \alpha W-[D, \bar{D}] T-\frac{3}{c} T^{2}\right](w) \\
& +\frac{1}{(z-w)}\left[\alpha \partial W-\frac{1}{2} \partial[D, \bar{D}] T-\frac{3}{c} \partial T T\right](w)+\cdots
\end{aligned}
$$

Also we take the large $c$ limit. In section 2, we introduced a new stress energy tensor by looking at the second-order pole. One easily sees the relative coefficient $\frac{1}{2}$ between the second-order and the first-order singular terms from the conformal invariance.

The spin-2 and spin- $\frac{5}{2}$ operator product expansions are

$$
\begin{aligned}
& W(z)(D W \pm \bar{D} W)(w)=\frac{1}{(z-w)^{3}} 3[D T \mp \bar{D} T](w) \\
& +\frac{1}{(z-w)^{2}}\left[\alpha(D W \pm \bar{D} W)+\frac{c}{(-1+c)} \partial(D T \mp \bar{D} T)-\frac{3}{(-1+c)} T(D T \pm \bar{D} T)\right](w) \\
& +\frac{1}{(z-w)}\left[(D V \mp \bar{D} V)+\frac{2\left(-27+12 c+c^{2}\right)}{(3+c)(-12+5 c)} \alpha \partial(D W \pm \bar{D} W)\right. \\
& \mp \frac{c\left(63-9 c+2 c^{2}\right)}{4(-1+c)(6+c)(-3+2 c)} \partial^{2} \bar{D} T-\frac{6(-15+c)}{(3+c)(-12+5 c)} \alpha T(D W \mp \bar{D} W) \\
& -\frac{9(3+4 c)}{(-1+c)(6+c)(-3+2 c)} T T(D T \mp \bar{D} T)+\frac{54(-1+c)}{(3+c)(-12+5 c)} \alpha(D T \mp \bar{D} T) W
\end{aligned}
$$

\footnotetext{
${ }^{11}$ With the $\mathcal{N}=2$ package [40], one obtains the following component operator product expansions automatically by using the command "N2OPEToComponents".
} 


$$
\begin{aligned}
& -\frac{27 c}{2(6+c)(-3+2 c)}([D, \bar{D}] T D T \mp \bar{D} T[D, \bar{D}] T)+\frac{(-15+c)(-3+c) c}{2(-1+c)(6+c)(-3+2 c)} \partial^{2} D T \\
& -\frac{3(-15+c) c}{(-1+c)(6+c)(-3+2 c)}(\partial D T \pm \partial \bar{D} T) T \\
& \left.-\frac{3\left(-54+39 c+c^{2}\right)}{2(-1+c)(6+c)(-3+2 c)} \partial T(D T \pm \bar{D} T)\right](w)+\cdots \\
& \longrightarrow \frac{1}{(z-w)^{3}} 3[D T \mp \bar{D} T](w) \\
& +\frac{1}{(z-w)^{2}}\left[\alpha(D W \pm \bar{D} W)+\partial(D T \mp \bar{D} T)-\frac{3}{c} T(D T \pm \bar{D} T)\right](w) \\
& +\frac{1}{(z-w)}\left[(D V \mp \bar{D} V)+\frac{2}{5} \alpha \partial(D W \pm \bar{D} W) \mp \frac{1}{4} \partial^{2} \bar{D} T-\frac{6}{5 c} \alpha T(D W \mp \bar{D} W)\right. \\
& -\frac{18}{c^{2}} T T(D T \mp \bar{D} T)+\frac{54}{5 c} \alpha(D T \mp \bar{D} T) W-\frac{27}{4 c}([D, \bar{D}] T D T \mp \bar{D} T[D, \bar{D}] T) \\
& \left.-\frac{3}{2 c}(\partial D T \pm \partial \bar{D} T) T-\frac{3}{4 c} \partial T(D T \pm \bar{D} T)+\frac{1}{4} \partial^{2} D T\right](w)+\cdots .
\end{aligned}
$$

One realizes that the symmetry behind the two types of combination in the second- and third-components of $W(w)$ occurs in the right hand side except some derivative terms but at the classical level these derivative terms also can be combined.

The operator product expansion between the spin- 2 and spin-3 is given by

$$
\begin{aligned}
& W(z)(-1) \frac{1}{2}[D, \bar{D}] W(w)=\frac{1}{(z-w)^{4}} 3 T(w) \\
& +\frac{1}{(z-w)^{2}}\left[-\frac{3(-8+c)}{2(-12+5 c)} \alpha[D, \bar{D}] W-\frac{9 c(-12+5 c)}{4(-1+c)(6+c)(-3+2 c)} \partial[D, \bar{D}] T\right. \\
& +\frac{42}{(-12+5 c)} \alpha T W-\frac{3\left(36-9 c+8 c^{2}\right)}{2(-1+c)(6+c)(-3+2 c)} T[D, \bar{D}] T \\
& -\frac{9(3+4 c)}{(-1+c)(6+c)(-3+2 c)} T^{3}-\frac{9 c(-12+5 c)}{(-1+c)(6+c)(-3+2 c)} \bar{D} T D T \\
& \left.-\frac{3 c(-12+5 c)}{2(-1+c)(6+c)(-3+2 c)} \partial^{2} T+3 V\right](w) \\
& +\frac{1}{(z-w)}\left[-\frac{\left(-72+15 c+c^{2}\right)}{2(3+c)(-12+5 c)} \alpha \partial[D, \bar{D}] W+\frac{6(3+4 c)}{(3+c)(-12+5 c)} \alpha T \partial W\right. \\
& -\frac{6}{(3+c)} \alpha(\bar{D} T D W+D T \bar{D} W)-\frac{3 c(-12+5 c)}{(-1+c)(6+c)(-3+2 c)}(\partial \bar{D} T D T-\partial D T \bar{D} T) \\
& -\frac{3 c(3+4 c)}{2(-1+c)(6+c)(-3+2 c)} \partial[D, \bar{D}] T T-\frac{6(-15+c)}{(3+c)(-12+5 c)} \alpha \partial T W \\
& +\frac{9(-12+5 c)}{2(-1+c)(6+c)(-3+2 c)} \partial T[D, \bar{D}] T-\frac{9(3+4 c)}{(-1+c)(6+c)(-3+2 c)} \partial T T T
\end{aligned}
$$




$$
\begin{aligned}
& \left.-\frac{c(-6+13 c)}{4(-1+c)(6+c)(-3+2 c)} \partial^{3} T+\partial V\right](w)+\cdots \\
& \longrightarrow \frac{1}{(z-w)^{4}} 3 T(w) \\
& +\frac{1}{(z-w)^{2}}\left[-\frac{3}{10} \alpha[D, \bar{D}] W+\frac{42}{5 c} \alpha T W-\frac{6}{c} T[D, \bar{D}] T-\frac{18}{c^{2}} T^{3}-\frac{45}{2 c} \bar{D} T D T+3 V\right](w) \\
& +\frac{1}{(z-w)}\left[-\frac{1}{10} \alpha \partial[D, \bar{D}] W+\frac{24}{5 c} \alpha T \partial W-\frac{6}{c} \alpha(\bar{D} T D W+D T \bar{D} W)\right. \\
& \left.-\frac{15}{2 c}(\partial \bar{D} T D T-\partial D T \bar{D} T)-\frac{3}{c} \partial[D, \bar{D}] T T-\frac{6}{5 c} \alpha \partial T W-\frac{18}{c^{2}} \partial T T T+\partial V\right](w) \\
& +\cdots
\end{aligned}
$$

Note that the spin-3 field $V(w)$ and its descendant field $\partial V(w)$ arise here. In the $c \rightarrow \infty$ limit, the terms $\partial[D, \bar{D}] T(w), \partial^{2} T(w), \partial T[D, \bar{D}] T(w)$, and $\partial^{3} T(w)$ are vanishing with an appropriate limiting procedure.

The operator product expansion of spin- $\frac{5}{2}$ and spin $-\frac{5}{2}$ occurs as follows:

$$
\begin{aligned}
& D W(z) D W(w)=\frac{1}{(z-w)}\left[-\frac{12}{3+c} \alpha D T D W-\frac{6}{(-1+c)} \partial D T D T\right](w)+\cdots \\
& \longrightarrow \frac{1}{(z-w)}\left[-\frac{12}{c} \alpha D T D W-\frac{6}{c} \partial D T D T\right](w)+\cdots
\end{aligned}
$$

There are no any linear terms in the right hand side. At the linear level, there were no singular terms [16]. Similarly, one has the following operator product expansion

$$
\begin{aligned}
& D W(z) \bar{D} W(w)+\bar{D} W(z) D W(w)=\frac{1}{(z-w)^{5}} 2 c \\
& +\frac{1}{(z-w)^{3}}\left[4 \alpha W-\frac{(-3+5 c)}{(-1+c)}[D, \bar{D}] T-\frac{6}{(-1+c)} T^{2}\right](w) \\
& +\frac{1}{(z-w)^{2}}\left[2 \alpha \partial W-\frac{(-3+5 c)}{2(-1+c)} \partial[D, \bar{D}] T-\frac{6}{(-1+c)} \partial T T\right](w) \\
& +\frac{1}{(z-w)}\left[\frac{3(-6+c)(-1+c)}{(3+c)(-12+5 c)} \alpha \partial^{2} W-\frac{3 c\left(9-3 c+c^{2}\right)}{2(-1+c)(6+c)(-3+2 c)} \partial^{2}[D, \bar{D}] T\right. \\
& +\frac{6(-15+c)}{(3+c)(-12+5 c)} \alpha T[D, \bar{D}] W+\frac{9(3+4 c)}{(-1+c)(6+c)(-3+2 c)} T T[D, \bar{D}] T \\
& -\frac{36(3+4 c)}{(-1+c)(6+c)(-3+2 c)} T \bar{D} T D T+\frac{12(3+4 c)}{(3+c)(-12+5 c)} \alpha(\bar{D} T D W-D T \bar{D} W) \\
& +\frac{3\left(18-51 c+5 c^{2}\right)}{(-1+c)(6+c)(-3+2 c)}(\partial \bar{D} T D T+\partial D T \bar{D} T)-\frac{54(-1+c)}{(3+c)(-12+5 c)} \alpha[D, \bar{D}] T W \\
& +\frac{27 c}{2(6+c)(-3+2 c)}[D, \bar{D}] T[D, \bar{D}] T-\frac{9(3+4 c)}{(-1+c)(6+c)(-3+2 c)} \partial[D, \bar{D}] T T
\end{aligned}
$$




$$
\begin{aligned}
& -\frac{9(-6+c)}{2(6+c)(-3+2 c)} \partial T \partial T-\frac{3\left(-18+24 c+c^{2}\right)}{(-1+c)(6+c)(-3+2 c)} \partial^{2} T T \\
& \left.-\frac{3(3+4 c)}{(-1+c)(6+c)(-3+2 c)} \partial^{3} T-[D, \bar{D}] V\right](w)+\cdots \\
& \longrightarrow \frac{1}{(z-w)^{5}} 2 c \\
& +\frac{1}{(z-w)^{3}}\left[4 \alpha W-5[D, \bar{D}] T-\frac{6}{c} T^{2}\right](w)+\frac{1}{(z-w)^{2}}\left[2 \alpha \partial W-\frac{5}{2} \partial[D, \bar{D}] T-\frac{6}{c} \partial T T\right](w) \\
& +\frac{1}{(z-w)}\left[\frac{3}{5} \alpha \partial^{2} W-\frac{3}{4} \partial^{2}[D, \bar{D}] T+\frac{6}{5 c} \alpha T[D, \bar{D}] W+\frac{18}{c^{2}} T T[D, \bar{D}] T\right. \\
& -\frac{72}{c^{2}} T \bar{D} T D T+\frac{48}{5 c} \alpha(\bar{D} T D W-D T \bar{D} W)+\frac{15}{2 c}(\partial \bar{D} T D T+\partial D T \bar{D} T) \\
& \left.-\frac{54}{5 c} \alpha[D, \bar{D}] T W+\frac{27}{4 c}[D, \bar{D}] T[D, \bar{D}] T-\frac{9}{4 c} \partial T \partial T-\frac{3}{2 c} \partial^{2} T T-[D, \bar{D}] V\right](w)+\cdots,(\mathrm{C} .5)
\end{aligned}
$$

where the spin-4 field occurs in the right hand side. In the $c \rightarrow \infty$ limit, the terms $\partial[D, \bar{D}] T T(w)$ and $\partial^{3} T(w)$ are vanishing. One can easily analyze the relative coefficients appearing in the spin-2 fields and its descendant fields.

The different combination between the spin- $\frac{5}{2}$ fields leads to

$$
\begin{aligned}
& D W(z) \bar{D} W(w)-\bar{D} W(z) D W(w)=\frac{1}{(z-w)^{4}} 6 T(w)+\frac{1}{(z-w)^{3}} 3 \partial T(w) \\
& +\frac{1}{(z-w)^{2}}\left[\frac{2(6+c)}{(-12+5 c)} \alpha[D, \bar{D}] W+\frac{2 c\left(9-3 c+c^{2}\right)}{(-1+c)(6+c)(-3+2 c)} \partial^{2} T\right. \\
& -\frac{3(-3+c)(-6+13 c)}{2(-1+c)(6+c)(-3+2 c)} \partial[D, \bar{D}] T+\frac{84}{(-12+5 c)} \alpha T W \\
& -\frac{6\left(9+5 c^{2}\right)}{(-1+c)(6+c)(-3+2 c)} T[D, \bar{D}] T-\frac{18(3+4 c)}{(-1+c)(6+c)(-3+2 c)} T^{3} \\
& \left.-\frac{6(-3+c)(-6+13 c)}{(-1+c)(6+c)(-3+2 c)} \bar{D} T D T+6 V\right](w) \\
& +\frac{1}{(z-w)}\left[\frac{(6+c)}{(-12+5 c)} \alpha \partial[D, \bar{D}] W+\frac{\left(-18+9 c-13 c^{2}+c^{3}\right)}{2(-1+c)(6+c)(-3+2 c)} \partial^{3} T\right. \\
& +\frac{42}{(-12+5 c)} \alpha \partial(T W)-\frac{3(-3+c)(-6+13 c)}{(-1+c)(6+c)(-3+2 c)}(\partial \bar{D} T D T-\partial D T \bar{D} T) \\
& -\frac{3\left(9+5 c^{2}\right)}{(-1+c)(6+c)(-3+2 c)}(\partial[D, \bar{D}] T T+\partial T[D, \bar{D}] T) \\
& \left.-\frac{27(3+4 c)}{(-1+c)(6+c)(-3+2 c)} \partial T T T+3 \partial V\right](w)+\cdots \\
& \longrightarrow \frac{1}{(z-w)^{4}} 6 T(w)+\frac{1}{(z-w)^{3}} 3 \partial T(w)
\end{aligned}
$$




$$
\begin{aligned}
& +\frac{1}{(z-w)^{2}}\left[\frac{2}{5} \alpha[D, \bar{D}] W+\partial^{2} T+\frac{84}{5 c} \alpha T W-\frac{15}{c} T[D, \bar{D}] T-\frac{36}{c^{2}} T^{3}\right. \\
& \left.-\frac{39}{c} \bar{D} T D T+6 V\right](w) \\
& +\frac{1}{(z-w)}\left[\frac{1}{5} \alpha \partial[D, \bar{D}] W+\frac{1}{4} \partial^{3} T+\frac{42}{5 c} \alpha \partial(T W)-\frac{39}{2 c}(\partial \bar{D} T D T-\partial D T \bar{D} T)\right. \\
& \left.-\frac{15}{2 c}(\partial[D, \bar{D}] T T+\partial T[D, \bar{D}] T)-\frac{54}{c^{2}} \partial T T T+3 \partial V\right](w)+\cdots
\end{aligned}
$$

In this case, the spin-3 field $V(w)$ and its descendant field $\partial V(w)$ appear. In the $c \rightarrow \infty$ limit, the term $\partial[D, \bar{D}] T(w)$ vanishes. By adding or subtracting the two equations (C.5) and (C.6), one obtains $D W(z) \bar{D} W(w)$ and $\bar{D} W(z) D W(w)$ independently.

One also has the following operator product expansion without any linear terms

$$
\begin{aligned}
& \bar{D} W(z) \bar{D} W(w)=\frac{1}{(z-w)}\left[\frac{12}{3+c} \alpha \bar{D} T \bar{D} W-\frac{6}{(-1+c)} \partial \bar{D} T \bar{D} T\right](w)+\cdots \\
& \longrightarrow \frac{1}{(z-w)}\left[\frac{12}{c} \alpha \bar{D} T \bar{D} W-\frac{6}{c} \partial \bar{D} T \bar{D} T\right](w)+\cdots
\end{aligned}
$$

There were no singular terms at the linear level [16].

The next operator product expansion between the spin- $\frac{5}{2}$ and the spin-3 leads to

$$
\begin{aligned}
& (D W \pm \bar{D} W)(z)(-1) \frac{1}{2}[D, \bar{D}] W(w)=\frac{1}{(z-w)^{4}} \frac{15}{2}[D T \pm \bar{D} T](w) \\
& +\frac{1}{(z-w)^{3}}\left[\alpha(D W \mp \bar{D} W)+\frac{(-3+5 c)}{2(-1+c)} \partial(D T \pm \bar{D} T)-\frac{3}{(-1+c)} T(D T \mp \bar{D} T)\right](w) \\
& +\frac{1}{(z-w)^{2}}\left[\frac{\left(-9+21 c+2 c^{2}\right)}{(3+c)(-12+5 c)} \alpha \partial(D W \mp \bar{D} W)+\frac{(-3+c)\left(18-114 c+5 c^{2}\right)}{4(-1+c)(6+c)(-3+2 c)} \partial^{2} D T\right. \\
& \pm \frac{\left(108+423 c-93 c^{2}+10 c^{3}\right)}{8(-1+c)(6+c)(-3+2 c)} \partial^{2} \bar{D} T+\frac{3(57+13 c)}{(3+c)(-12+5 c)} \alpha T(D W \pm \bar{D} W) \\
& -\frac{63(3+4 c)}{2(-1+c)(6+c)(-3+2 c)}(T T D T \pm T T \bar{D} T)+\frac{3(33+23 c)}{(3+c)(-12+5 c)} \alpha(D T \pm \bar{D} T) W \\
& -\frac{3\left(72-99 c+55 c^{2}\right)}{4(-1+c)(6+c)(-3+2 c)}([D, \bar{D}] T D T \pm \bar{D} T[D, \bar{D}] T) \\
& -\frac{3\left(-36-87 c+11 c^{2}\right)}{2(-1+c)(6+c)(-3+2 c)}(\partial D T \mp \partial \bar{D} T) T \\
& \left.+\frac{3\left(90-129 c+25 c^{2}\right)}{4(-1+c)(6+c)(-3+2 c)} \partial T(D T \mp \bar{D} T)+\frac{7}{2}(D V \pm \bar{D} V)\right](w) \\
& +\frac{1}{(z-w)}\left[\frac{(6+c)}{2(-12+5 c)} \alpha \partial^{2}(D W \mp \bar{D} W)+\frac{(-15+c)(-3+c) c}{4(-1+c)(6+c)(-3+2 c)} \partial^{3}(D T \pm \bar{D} T)\right.
\end{aligned}
$$




$$
\begin{aligned}
& +\frac{21}{(-12+5 c)} \alpha T(D W \pm \partial \bar{D} W)+\frac{3}{(3+c)} \alpha(D T \mp \bar{D} T)[D, \bar{D}] W \\
& +\frac{9(3+4 c)}{(3+c)(-12+5 c)} \alpha(D T \pm \bar{D} T) \partial W+\frac{21}{(-12+5 c)} \alpha(\partial D T \pm \partial \bar{D} T) W \\
& -\frac{3\left(72-63 c+19 c^{2}\right)}{4(-1+c)(6+c)(-3+2 c)}(\partial D T \pm \partial \bar{D} T)[D, \bar{D}] T \\
& -\frac{27(3+4 c)}{2(-1+c)(6+c)(-3+2 c)}(\partial D T \pm \partial \bar{D} T) T T+\frac{3(3+7 c)}{4(-1+c)(-3+2 c)}(\partial D T \mp \partial \bar{D} T) \partial T \\
& -\frac{3\left(9+5 c^{2}\right)}{2(-1+c)(6+c)(-3+2 c)}\left(\partial^{2} D T \mp \partial^{2} \bar{D} T\right) T-\frac{3}{(3+c)} \alpha[D, \bar{D}] T(D W \mp \bar{D} W) \\
& -\frac{81 c}{4(6+c)(-3+2 c)} \partial[D, \bar{D}] T(D T \pm \bar{D} T)+\frac{3(33+2 c)}{(3+c)(-12+5 c)} \alpha(\partial T D W \pm \partial T \bar{D} W) \\
& -\frac{27(3+4 c)}{(-1+c)(6+c)(-3+2 c)} \partial T T(D T \pm \bar{D} T) \\
& \left.+\frac{27(-6+c)}{4(6+c)(-3+2 c)} \partial^{2} T(D T \mp \bar{D} T)+\frac{3}{2} \partial(D V \pm \bar{D} V)\right](w)+\cdots \\
& \longrightarrow \frac{1}{(z-w)^{4}} \frac{15}{2}[D T \pm \bar{D} T](w) \\
& +\frac{1}{(z-w)^{3}}\left[\alpha(D W \mp \bar{D} W)+\frac{5}{2} \partial(D T \pm \bar{D} T)-\frac{3}{c} T(D T \mp \bar{D} T)\right](w) \\
& +\frac{1}{(z-w)^{2}}\left[\frac{2}{5} \alpha \partial(D W \mp \bar{D} W)+\frac{5}{8} \partial^{2} D T \pm \frac{5}{8} \partial^{2} \bar{D} T+\frac{39}{5 c} \alpha T(D W \pm \bar{D} W)\right. \\
& -\frac{63}{c^{2}}(T T D T \pm T T \bar{D} T)+\frac{69}{5 c} \alpha(D T \pm \bar{D} T) W-\frac{165}{8 c}([D, \bar{D}] T D T \pm \bar{D} T[D, \bar{D}] T) \\
& \left.-\frac{33}{4 c}(\partial D T \mp \partial \bar{D} T) T+\frac{75}{8 c} \partial T(D T \mp \bar{D} T)+\frac{7}{2}(D V \pm \bar{D} V)\right](w) \\
& +\frac{1}{(z-w)}\left[\frac{1}{10} \alpha \partial^{2}(D W \mp \bar{D} W)+\frac{1}{8} \partial^{3}(D T \pm \bar{D} T)+\frac{21}{5 c} \alpha T(D W \pm \partial \bar{D} W)\right. \\
& +\frac{3}{c} \alpha(D T \mp \bar{D} T)[D, \bar{D}] W+\frac{36}{5 c} \alpha(D T \pm \bar{D} T) \partial W+\frac{21}{5 c} \alpha(\partial D T \pm \partial \bar{D} T) W \\
& -\frac{57}{8 c}(\partial D T \pm \partial \bar{D} T)[D, \bar{D}] T-\frac{27}{c^{2}}(\partial D T \pm \partial \bar{D} T) T T+\frac{21}{8 c}(\partial D T \mp \partial \bar{D} T) \partial T \\
& -\frac{15}{4 c}\left(\partial^{2} D T \mp \partial^{2} \bar{D} T\right) T-\frac{3}{c} \alpha[D, \bar{D}] T(D W \mp \bar{D} W)-\frac{81}{8 c} \partial[D, \bar{D}] T(D T \pm \bar{D} T) \\
& +\frac{6}{5 c} \alpha(\partial T D W \pm \partial T \bar{D} W)-\frac{54}{c^{2}} \partial T T(D T \pm \bar{D} T) \\
& \left.+\frac{27}{8 c} \partial^{2} T(D T \mp \bar{D} T)+\frac{3}{2} \partial(D V \pm \bar{D} V)\right](w)+\cdots .
\end{aligned}
$$

The spin- $\frac{7}{2}$ field and its descendant field appear in the right hand side. At the classical limit, the symmetry between $D$ and $\bar{D}$ which act on the fields is manifest at both sides. At the 
quantum level, the two derivative terms do not have common factors.

Also one has, from (C.8),

$$
\begin{aligned}
& (-1) \frac{1}{2}[D, \bar{D}] W(z)(D W \pm \bar{D} W)(w)=\frac{1}{(z-w)^{4}} \frac{15}{2}[D T \pm \bar{D} T](w) \\
& +\frac{1}{(z-w)^{3}}\left[-\alpha(D W \mp \bar{D} W)+\frac{(-6+5 c)}{(-1+c)} \partial(D T \pm \bar{D} T)+\frac{3}{(-1+c)} T(D T \mp \bar{D} T)\right](w) \\
& +\frac{1}{(z-w)^{2}}\left[-\frac{3\left(-9-6 c+c^{2}\right)}{(3+c)(-12+5 c)} \alpha \partial(D W \mp \bar{D} W)+\frac{3 c\left(81-30 c+5 c^{2}\right)}{4(-1+c)(6+c)(-3+2 c)} \partial^{2} D T\right. \\
& \pm \frac{3\left(72+63 c-5 c^{2}+10 c^{3}\right)}{8(-1+c)(6+c)(-3+2 c)} \partial^{2} \bar{D} T+\frac{3(57+13 c)}{(3+c)(-12+5 c)} \alpha T(D W \pm \bar{D} W) \\
& -\frac{63(3+4 c)}{2(-1+c)(6+c)(-3+2 c)}(T T D T \pm T T \bar{D} T)+\frac{3(33+23 c)}{(3+c)(-12+5 c)} \alpha(D T \pm \bar{D} T) W \\
& -\frac{3\left(72-99 c+55 c^{2}\right)}{4(-1+c)(6+c)(-3+2 c)}([D, \bar{D}] T D T \pm \bar{D} T[D, \bar{D}] T) \\
& -\frac{21(-15+c) c}{2(-1+c)(6+c)(-3+2 c)}(\partial D T \mp \partial \bar{D} T) T \\
& \left.+\frac{9\left(6-31 c+11 c^{2}\right)}{4(-1+c)(6+c)(-3+2 c)} \partial T(D T \mp \bar{D} T)+\frac{7}{2}(D V \pm \bar{D} V)\right](w) \\
& +\frac{1}{(z-w)}\left[-\frac{(-15+c) c}{(3+c)(-12+5 c)} \alpha \partial^{2}(D W \mp \bar{D} W)+\frac{c\left(9-3 c+c^{2}\right)}{(-1+c)(6+c)(-3+2 c)} \partial^{3}(D T \pm \bar{D} T)\right. \\
& +\frac{18(6+c)}{(3+c)(-12+5 c)} \alpha T(D W \pm \partial \bar{D} W)-\frac{3}{(3+c)} \alpha(D T \mp \bar{D} T)[D, \bar{D}] W \\
& +\frac{3(24+11 c)}{(3+c)(-12+5 c)} \alpha(D T \pm \bar{D} T) \partial W+\frac{12(3+4 c)}{(3+c)(-12+5 c)} \alpha(\partial D T \pm \partial \bar{D} T) W \\
& -\frac{27 c}{(6+c)(-3+2 c)}(\partial D T \pm \partial \bar{D} T)[D, \bar{D}] T \\
& -\frac{18(3+4 c)}{(-1+c)(6+c)(-3+2 c)}(\partial D T \pm \partial \bar{D} T) T T+\frac{3(3+c)}{(-1+c)(-3+2 c)}(\partial D T \mp \partial \bar{D} T) \partial T \\
& -\frac{6\left(9-3 c+c^{2}\right)}{(-1+c)(6+c)(-3+2 c)}\left(\partial^{2} D T \mp \partial^{2} \bar{D} T\right) T+\frac{3}{(3+c)} \alpha[D, \bar{D}] T(D W \mp \bar{D} W) \\
& -\frac{3\left(18-18 c+7 c^{2}\right)}{(-1+c)(6+c)(-3+2 c)} \partial[D, \bar{D}] T(D T \pm \bar{D} T)+\frac{3(24+11 c)}{(3+c)(-12+5 c)} \alpha(\partial T D W \pm \partial T \bar{D} W) \\
& -\frac{36(3+4 c)}{(-1+c)(6+c)(-3+2 c)} \partial T T(D T \pm \bar{D} T) \\
& \left.+\frac{3 c(-12+5 c)}{(-1+c)(6+c)(-3+2 c)} \partial^{2} T(D T \mp \bar{D} T)+2 \partial(D V \pm \bar{D} V)\right](w)+\cdots
\end{aligned}
$$




$$
\begin{aligned}
& \longrightarrow \frac{1}{(z-w)^{4}} \frac{15}{2}[D T \pm \bar{D} T](w) \\
& +\frac{1}{(z-w)^{3}}\left[-\alpha(D W \mp \bar{D} W)+5 \partial(D T \pm \bar{D} T)+\frac{3}{c} T(D T \mp \bar{D} T)\right](w) \\
& +\frac{1}{(z-w)^{2}}\left[-\frac{3}{5} \alpha \partial(D W \mp \bar{D} W)+\frac{15}{8} \partial^{2} D T \pm \frac{15}{8} \partial^{2} \bar{D} T+\frac{39}{5 c} \alpha T(D W \pm \bar{D} W)\right. \\
& -\frac{63}{c^{2}}(T T D T \pm T T \bar{D} T)+\frac{69}{5 c} \alpha(D T \pm \bar{D} T) W-\frac{165}{8 c}([D, \bar{D}] T D T \pm \bar{D} T[D, \bar{D}] T) \\
& \left.-\frac{21}{4 c}(\partial D T \mp \partial \bar{D} T) T+\frac{99}{8 c} \partial T(D T \mp \bar{D} T)+\frac{7}{2}(D V \pm \bar{D} V)\right](w) \\
& +\frac{1}{(z-w)}\left[-\frac{1}{5} \alpha \partial^{2}(D W \mp \bar{D} W)+\frac{1}{2} \partial^{3}(D T \pm \bar{D} T)+\frac{18}{5 c} \alpha T(D W \pm \partial \bar{D} W)\right. \\
& -\frac{3}{c} \alpha(D T \mp \bar{D} T)[D, \bar{D}] W+\frac{33}{5 c} \alpha(D T \pm \bar{D} T) \partial W+\frac{48}{5 c} \alpha(\partial D T \pm \partial \bar{D} T) W \\
& -\frac{27}{2 c}(\partial D T \pm \partial \bar{D} T)[D, \bar{D}] T-\frac{36}{c^{2}}(\partial D T \pm \partial \bar{D} T) T T+\frac{3}{2 c}(\partial D T \mp \partial \bar{D} T) \partial T \\
& -\frac{3}{c}\left(\partial^{2} D T \mp \partial^{2} \bar{D} T\right) T+\frac{3}{c} \alpha[D, \bar{D}] T(D W \mp \bar{D} W)-\frac{21}{2 c} \partial[D, \bar{D}] T(D T \pm \bar{D} T) \\
& +\frac{33}{5 c} \alpha(\partial T D W \pm \partial T \bar{D} W)-\frac{72}{c^{2}} \partial T T(D T \pm \bar{D} T) \\
& \left.+\frac{15}{2 c} \partial^{2} T(D T \mp \bar{D} T)+2 \partial(D V \pm \bar{D} V)\right](w)+\cdots
\end{aligned}
$$

Finally, the spin-3 and spin-3 operator product expansion has the following form

$$
\begin{aligned}
& (-1) \frac{1}{2}[D, \bar{D}] W(z)(-1) \frac{1}{2}[D, \bar{D}] W(w)=\frac{1}{(z-w)^{6}} \frac{5}{2} c \\
& +\frac{1}{(z-w)^{4}}\left[3 \alpha W-\frac{3(-4+5 c)}{2(-1+c)}[D, \bar{D}] T-\frac{9}{2(-1+c)} T^{2}\right](w) \\
& +\frac{1}{(z-w)^{3}}\left[\frac{3}{2} \alpha \partial W-\frac{3(-4+5 c)}{4(-1+c)} \partial[D, \bar{D}] T-\frac{9}{2(-1+c)} \partial T T\right](w) \\
& +\frac{1}{(z-w)^{2}}\left[\frac{3\left(-12-19 c+3 c^{2}\right)}{4(3+c)(-12+5 c)} \alpha \partial^{2} W-\frac{9 c\left(30-11 c+2 c^{2}\right)}{8(-1+c)(6+c)(-3+2 c)} \partial^{2}[D, \bar{D}] T\right. \\
& -\frac{18(6+c)}{(3+c)(-12+5 c)} \alpha T[D, \bar{D}] W+\frac{18(3+4 c)}{(-1+c)(6+c)(-3+2 c)}(T T[D, \bar{D}] T-\partial[D, \bar{D}] T T) \\
& -\frac{72(3+4 c)}{(-1+c)(6+c)(-3+2 c)} T \bar{D} T D T+\frac{6(24+11 c)}{(3+c)(-12+5 c)} \alpha(\bar{D} T D W-D T \bar{D} W) \\
& +\frac{42(-6+c) c}{(-1+c)(6+c)(-3+2 c)}(\partial \bar{D} T D T+\partial D T \bar{D} T)-\frac{12(3+4 c)}{(3+c)(-12+5 c)} \alpha[D, \bar{D}] T W \\
& +\frac{3\left(18-27 c+16 c^{2}\right)}{2(-1+c)(6+c)(-3+2 c)}[D, \bar{D}] T[D, \bar{D}] T \\
& -\frac{9\left(6-19 c+6 c^{2}\right)}{4(-1+c)(6+c)(-3+2 c)} \partial T \partial T+\frac{9\left(6-43 c+2 c^{2}\right)}{4(-1+c)(6+c)(-3+2 c)} \partial^{2} T T
\end{aligned}
$$




$$
\begin{aligned}
& \left.-\frac{6(3+4 c)}{(-1+c)(6+c)(-3+2 c)} \partial^{3} T-2[D, \bar{D}] V\right](w) \\
& +\frac{1}{(z-w)}\left[\frac{(-15+c) c}{2(3+c)(-12+5 c)} \alpha \partial^{3} W-\frac{c\left(30-11 c+2 c^{2}\right)}{4(-1+c)(6+c)(-3+2 c)} \partial^{3}[D, \bar{D}] T\right. \\
& -\frac{9(6+c)}{(3+c)(-12+5 c)} \alpha \partial(T[D, \bar{D}] W)+\frac{3(24+11 c)}{(3+c)(-12+5 c)} \alpha(\bar{D} T \partial D W+\partial \bar{D} T D W) \\
& -\frac{36(3+4 c)}{(-1+c)(6+c)(-3+2 c)}(\partial \bar{D} T T D T-\partial D T T \bar{D} T) \\
& +\frac{3\left(18-18 c+7 c^{2}\right)}{(-1+c)(6+c)(-3+2 c)} \partial^{2} \bar{D} T D T-\frac{6(3+4 c)}{(3+c)(-12+5 c)} \alpha \partial([D, \bar{D}] T W) \\
& +\frac{3\left(18-27 c+16 c^{2}\right)}{2(-1+c)(6+c)(-3+2 c)} \partial[D, \bar{D}] T[D, \bar{D}] T-\frac{3(24+11 c)}{(3+c)(-12+5 c)} \alpha \partial(D T \bar{D} W) \\
& +\frac{9(3+4 c)}{(-1+c)(6+c)(-3+2 c)}(\partial[D, \bar{D}] T T T-\partial[D, \bar{D}] T \partial T) \\
& +\frac{36(3+4 c)}{(-1+c)(6+c)(-3+2 c)} \partial D T T \bar{D} T+\frac{3\left(18-18 c+7 c^{2}\right)}{(-1+c)(6+c)(-3+2 c)} \partial^{2} D T \bar{D} T \\
& +\frac{18(3+4 c)}{(-1+c)(6+c)(-3+2 c)} \partial T T[D, \bar{D}] T-\frac{9\left(6-c+2 c^{2}\right)}{2(-1+c)(6+c)(-3+2 c)} \partial^{2} T \partial T \\
& \left.+\frac{3\left(9-3 c+c^{2}\right)}{(-1+c)(6+c)(-3+2 c)} \partial^{3} T T-\frac{3(3+4 c)}{2(-1+c)(6+c)(-3+2 c)} \partial^{4} T-\partial[D, \bar{D}] V\right](w)+\cdots \\
& \longrightarrow \frac{1}{(z-w)^{6}} \frac{5}{2} c+\frac{1}{(z-w)^{4}}\left[3 \alpha W-\frac{15}{2}[D, \bar{D}] T-\frac{9}{2 c} T^{2}\right](w) \\
& +\frac{1}{(z-w)^{3}}\left[\frac{3}{2} \alpha \partial W-\frac{15}{4} \partial[D, \bar{D}] T-\frac{9}{2 c} \partial T T\right](w) \\
& +\frac{1}{(z-w)^{2}}\left[\frac{9}{20} \alpha \partial^{2} W-\frac{9}{8} \partial^{2}[D, \bar{D}] T-\frac{18}{5 c} \alpha T[D, \bar{D}] W+\frac{36}{c^{2}}(T T[D, \bar{D}] T-\partial[D, \bar{D}] T T)\right. \\
& -\frac{144}{c^{2}} T \bar{D} T D T+\frac{66}{5 c} \alpha(\bar{D} T D W-D T \bar{D} W)+\frac{21}{c}(\partial \bar{D} T D T+\partial D T \bar{D} T)-\frac{48}{5 c} \alpha[D, \bar{D}] T W \\
& \left.+\frac{12}{c}[D, \bar{D}] T[D, \bar{D}] T-\frac{27}{4 c} \partial T \partial T+\frac{9}{4 c} \partial^{2} T T-2[D, \bar{D}] V\right](w) \\
& +\frac{1}{(z-w)}\left[\frac{1}{10} \alpha \partial^{3} W-\frac{1}{4} \partial^{3}[D, \bar{D}] T-\frac{9}{5 c} \alpha \partial(T[D, \bar{D}] W)+\frac{33}{5 c} \alpha(\bar{D} T \partial D W+\partial \bar{D} T D W)\right. \\
& -\frac{72}{c^{2}}(\partial \bar{D} T T D T-\partial D T T \bar{D} T)+\frac{21}{2 c} \partial^{2} \bar{D} T D T-\frac{24}{5 c} \alpha \partial([D, \bar{D}] T W) \\
& +\frac{12}{c} \partial[D, \bar{D}] T[D, \bar{D}] T-\frac{33}{5 c} \alpha \partial(D T \bar{D} W)+\frac{18}{c^{2}}(\partial[D, \bar{D}] T T T-\partial[D, \bar{D}] T \partial T) \\
& +\frac{72}{c^{2}} \partial D T T \bar{D} T+\frac{21}{2 c} \partial^{2} D T \bar{D} T+\frac{36}{c^{2}} \partial T T[D, \bar{D}] T-\frac{9}{2 c} \partial^{2} T \partial T \\
& \left.+\frac{3}{2 c} \partial^{3} T T-\partial[D, \bar{D}] V\right](w)+\cdots \text {. }
\end{aligned}
$$


The spin-4 field and its descendant field appear in the right hand side. In the $c \rightarrow \infty$ limit, the term $\partial^{3} T(w)$ vanishes.

Of course, the classical limits (C.1) - C.10) where the central charge is given by (3.1) and the self-coupling constant is given by (3.2) can be combined into (3.3). For the fixed $N$ with large $c$ limit, the corresponding self-coupling constant behaves as before. In next Appendices, we list some relevant operator product expansions which will be necessary to transform the operator product expansions in the primary basis of this Appendix $C$ into those in the nonprimary basis of [13].

\section{Appendix D The remaining operator product expansions in the $A d S_{3}$ bulk theory: Nonprimary basis}

We have seen that the above relations (4.2), (4.3) and (4.4) provide the quantum and classical operator product expansions in the notation of [13]. In this Appendix $D$, we would like to complete the remaining ones.

The operator product expansion between the spin- $\frac{5}{2}$ and spin- $\frac{5}{2}$ (this does not change eventhough one introduces the modified stress energy tensor (2.21)), from (C.4), (C.5), and (C.7), is given by

$$
\begin{aligned}
& (D W+\bar{D} W)(z)(D W+\bar{D} W)(w)=\frac{1}{(z-w)^{5}} 2 c \\
& +\frac{1}{(z-w)^{3}}\left[4 \alpha W-\frac{(-3+5 c)}{(-1+c)}[D, \overline{\bar{D}}] T+\frac{9}{c} T^{2}\right](w) \\
& +\frac{1}{(z-w)^{2}}\left[2 \alpha \partial W-\frac{(-3+5 c)}{2(-1+c)} \partial[\widetilde{D, \bar{D}}] T+\frac{9}{c} \partial T T\right](w) \\
& +\frac{1}{(z-w)}\left[\frac{3(-6+c)(-1+c)}{(3+c)(-12+5 c)} \alpha \partial^{2} W-\frac{3 c\left(9-3 c+c^{2}\right)}{2(-1+c)(6+c)(-3+2 c)} \partial^{2}[\widetilde{D, \bar{D}}] T\right. \\
& +\frac{6(-15+c)}{(3+c)(-12+5 c)} \alpha T[D, \widetilde{\bar{D}}] W-\frac{9(-12+5 c)}{(-1+c)(6+c)(-3+2 c)} T T[D, \widetilde{\bar{D}}] T \\
& +\frac{18(51+5 c)}{c(3+c)(-12+5 c)} \alpha T T W+\frac{27(-15+c)}{2(-1+c) c(6+c)(-3+2 c)} T^{4} \\
& -\frac{36(3+4 c)}{(-1+c)(6+c)(-3+2 c)} T \bar{D} T D T+\frac{12(3+4 c)}{(3+c)(-12+5 c)} \alpha(\bar{D} T D W-D T \bar{D} W) \\
& +\frac{3\left(18-51 c+5 c^{2}\right)}{(-1+c)(6+c)(-3+2 c)}(\partial \bar{D} T D T+\partial D T \bar{D} T)-\frac{54(-1+c)}{(3+c)(-12+5 c)} \alpha[\widetilde{\bar{D}}] T W \\
& +\frac{27 c}{2(6+c)(-3+2 c)}[D, \widetilde{\bar{D}}] T[D, \widetilde{\bar{D}}] T-\frac{9(3+4 c)}{(-1+c)(6+c)(-3+2 c)} \partial[D, \widetilde{\bar{D}}] T T
\end{aligned}
$$




$$
\begin{aligned}
& -\frac{9(-6+c)}{2(6+c)(-3+2 c)} \partial T \partial T-\frac{3\left(63-57 c+c^{2}\right)}{(-1+c)(6+c)(-3+2 c)} \partial^{2} T T \\
& -\frac{12}{(3+c)} \alpha D T D W-\frac{6}{(-1+c)} \partial D T D T \\
& +\frac{12}{(3+c)} \alpha \bar{D} T \bar{D} W-\frac{6}{(-1+c)} \partial \bar{D} T \bar{D} T \\
& \left.+\frac{54(3+4 c)}{(-1+c) c(6+c)(-3+2 c)} \partial T T T-[D, \bar{D}] V\right](w)+\cdots \\
& \longrightarrow \frac{1}{(z-w)^{5}} 2 c+\frac{1}{(z-w)^{3}}\left[4 \alpha W-5[D, \bar{D}] T+\frac{9}{c} T^{2}\right](w) \\
& +\frac{1}{(z-w)^{2}}\left[2 \alpha \partial W-\frac{5}{2} \partial[\overline{\bar{D}}] T+\frac{9}{2 c} \partial T^{2}\right](w) \\
& +\frac{1}{(z-w)}\left[\frac{3}{5} \alpha \partial^{2} W-\frac{3}{4} \partial^{2}[\widetilde{\bar{D}}] T+\frac{6}{5 c} \alpha T[D, \widetilde{\bar{D}}] W-\frac{45}{2 c^{2}} T T[\widetilde{\bar{D}}] T\right. \\
& +\frac{18}{c^{2}} \alpha T T W+\frac{27}{4 c^{3}} T^{4}-\frac{72}{c^{2}} T \bar{D} T D T+\frac{48}{5 c} \alpha(\bar{D} T D W-D T \bar{D} W) \\
& +\frac{15}{2 c}(\partial \bar{D} T D T+\partial D T \bar{D} T)-\frac{54}{5 c} \alpha[D \overline{\bar{D}}] T W+\frac{27}{4 c}[D, \bar{D}] T[D, \bar{D}] T \\
& -\frac{9}{4 c} \partial T \partial T-\frac{3}{2 c} \partial^{2} T T-[D, \bar{D}] V-\frac{12}{c} \alpha D T D W-\frac{6}{c} \partial D T D T \\
& \left.+\frac{12}{c} \alpha \bar{D} T \bar{D} W-\frac{6}{c} \partial \bar{D} T \bar{D} T\right] \widetilde{(w)+\cdots}
\end{aligned}
$$

Except the terms $T \bar{D} T D T(w), \bar{D} T D W(w), D T \bar{D} W, D T D W)(w)$, and $\bar{D} T \bar{D} W(w)$, one can easily check that all the remaining fields appear in the bulk theory computations [13]. The relative coefficients in the spin- 2 field and its descendant fields can be obtained from the formula [48], $\frac{\Gamma[2+1]}{\Gamma[2]} \times \frac{\Gamma[4]}{\Gamma[4+1]}=\frac{1}{2}$ and $\frac{1}{2 !} \frac{\Gamma[2+2]}{\Gamma[2]} \times \frac{\Gamma[4]}{\Gamma[4+2]}=\frac{3}{20}$. That is, the coefficient 2 in the $\partial W(w)$ is obtained from $4 \times \frac{1}{2}=2$ and the coefficient $\frac{3}{5}$ in $\partial^{2} W(w)$ term is coming from $4 \times \frac{3}{20}=\frac{3}{5}$.

From the equations (C.4), (C.5), and (C.7), other type of spin- $\frac{5}{2}$ and spin- $\frac{5}{2}$ operator product expansion is

$$
\begin{aligned}
& (D W+\bar{D} W)(z)(D W-\bar{D} W)(w)=-\frac{1}{(z-w)^{4}} 6 T(w)-\frac{1}{(z-w)^{3}} 3 \partial T(w) \\
& -\frac{1}{(z-w)^{2}}\left[\frac{2(6+c)}{(-12+5 c)} \alpha[D, \widetilde{\bar{D}}] W+\frac{2 c\left(9-3 c+c^{2}\right)}{(-1+c)(6+c)(-3+2 c)} \partial^{2} T\right. \\
& -\frac{3(-3+c)(-6+13 c)}{2(-1+c)(6+c)(-3+2 c)} \partial[\widetilde{\widetilde{D}}] T+\frac{12}{c} \alpha T W \\
& -\frac{6\left(9+5 c^{2}\right)}{(-1+c)(6+c)(-3+2 c)} T[D, \widetilde{\bar{D}}] T+\frac{18\left(9-3 c+c^{2}\right)}{(-1+c) c(6+c)(-3+2 c)} T^{3}
\end{aligned}
$$




$$
\begin{aligned}
& \left.-\frac{6(-3+c)(-6+13 c)}{(-1+c)(6+c)(-3+2 c)} \bar{D} T D T+\frac{9(-3+c)(-6+13 c)}{(-1+c) c(6+c)(-3+2 c)} \partial T T+6 V\right](w) \\
& -\frac{1}{(z-w)}\left[\frac{(6+c)}{(-12+5 c)} \alpha \partial[D, \widetilde{\bar{D}}] W+\frac{c\left(9-3 c+c^{2}\right)}{2(-1+c)(6+c)(-3+2 c)} \partial^{3} T\right. \\
& +\frac{6}{c} \alpha \partial(T W)-\frac{3(-3+c)(-6+13 c)}{(-1+c)(6+c)(-3+2 c)}(\partial \bar{D} T D T-\partial D T \bar{D} T) \\
& -\frac{3\left(9+5 c^{2}\right)}{(-1+c)(6+c)(-3+2 c)}(\partial[D, \bar{D}] T T+\partial T[D, \bar{D}] T) \\
& +\frac{27\left(9-3 c+c^{2}\right)}{(-1+c) c(6+c)(-3+2 c)} \partial T T T+3 \partial V \\
& -\frac{12}{(3+c)} \alpha D T D W-\frac{6}{(-1+c)} \partial D T D T \\
& \left.-\frac{12}{(3+c)} \alpha \bar{D} T \bar{D} W+\frac{6}{(-1+c)} \partial \bar{D} T \bar{D} T\right](w)+\cdots \\
& -\frac{1}{(z-w)^{4}} 6 T(w)-\frac{1}{(z-w)^{3}} 3 \partial T(w) \\
& -\frac{1}{(z-w)^{2}}\left[\frac{2}{5} \alpha[D, \widetilde{\bar{D}}] W+\partial^{2} T+\frac{12}{c} \alpha T W-\frac{15}{c} T[\widetilde{\bar{D}}] T+\frac{9}{c^{2}} T^{3}\right. \\
& \left.-\frac{39}{c} \bar{D} T D T+6 V\right](w) \\
& -\frac{1}{(z-w)}\left[\frac{1}{5} \alpha \partial[D, \widetilde{\bar{D}}] W+\frac{1}{4} \partial^{3} T+\frac{6}{c} \alpha \partial(T W)-\frac{39}{2 c} \partial(\bar{D} T D T)\right. \\
& -\frac{15}{2 c} \partial(T[\widetilde{D} \bar{D}] T)+\frac{9}{2 c^{2}} \partial T^{3}+3 \partial V-\frac{12}{c} \alpha D T D W-\frac{6}{c} \partial D T D T \\
& \left.-\frac{12}{c} \alpha \bar{D} T \bar{D} W+\frac{6}{c} \partial \bar{D} T \bar{D} T\right](w)+\cdots
\end{aligned}
$$

The relative coefficients $\frac{12}{c}$ and $\frac{6}{c}$ in the $\alpha$-dependent terms provide the correct values in [13]. Also $\frac{15}{c}$ in the second order singular term and $\frac{15}{2 c}$ in the first order singular term have the correct behaviors. Furthermore, the coefficient $\frac{9}{c^{2}}$ in $T^{3}(w)$ term and the coefficient $\frac{27}{2 c^{2}}$ in its derivative term behave correctly as in [13]. There are also the terms $\bar{D} T D T(w), D T D W$, and $\bar{D} T \bar{D} W$. The relative coefficients $6,3,1$, and $\frac{1}{4}$ in the spin- 1 field and its descendant fields can be obtained from the coefficient formula in $[48,1]$.

From the equation ([C.9), the next operator product expansion between the spin-3 and the spin- $\frac{5}{2}$ has the following form

$$
\begin{aligned}
& (-1) \frac{1}{2}[D, \bar{D}] W(z)(D W \pm \bar{D} W)(w)=\frac{1}{(z-w)^{4}} \frac{3(-12+5 c)}{2 c}[D T \pm \bar{D} T](w) \\
& +\frac{1}{(z-w)^{3}}\left[-\frac{(6+c)}{c} \alpha(D W \mp \bar{D} W)+\frac{(-12+5 c)}{(-1+c)} \partial(D T \pm \bar{D} T)\right.
\end{aligned}
$$




$$
\begin{aligned}
& \left.-\frac{3(-12+5 c)}{(-1+c) c} T(D T \mp \bar{D} T)\right](w) \\
& +\frac{1}{(z-w)^{2}}\left[-\frac{3\left(-108+39 c-2 c^{2}+c^{3}\right)}{c(3+c)(-12+5 c)} \alpha \partial(D W \mp \bar{D} W)+\frac{3(-3+c)^{2}(-12+5 c)}{4(-1+c)(6+c)(-3+2 c)} \partial^{2} D T\right. \\
& \pm \frac{3\left(-756+882 c-303 c^{2}-29 c^{3}+10 c^{4}\right)}{8(-1+c) c(6+c)(-3+2 c)} \partial^{2} \bar{D} T+\frac{9\left(-36+21 c+c^{2}\right)}{c(3+c)(-12+5 c)} \alpha T(D W \pm \bar{D} W) \\
& +\frac{45\left(18-18 c+7 c^{2}\right)}{2(-1+c) c(6+c)(-3+2 c)}(T T D T \pm T T \bar{D} T)+\frac{3\left(108-75 c+23 c^{2}\right)}{c(3+c)(-12+5 c)} \alpha(D T \pm \bar{D} T) W \\
& -\frac{3(-12+5 c)(-15+11 c)}{4(-1+c)(6+c)(-3+2 c)}([D \widetilde{\bar{D}}] T D T \pm \bar{D} T[\widetilde{\overline{,} \bar{D}}] T) \\
& -\frac{9(-3+c)(-12+5 c)}{2(-1+c)(6+c)(-3+2 c)} \partial D T T \pm \frac{9\left(252-270 c+83 c^{2}+5 c^{3}\right)}{2(-1+c) c(6+c)(-3+2 c)} \partial \bar{D} T T \\
& -\frac{9\left(-144+234 c-137 c^{2}+5 c^{3}\right)}{4(-1+c) c(6+c)(-3+2 c)} \partial T D T \pm \frac{9(-12+5 c)\left(-30+19 c+c^{2}\right)}{4(-1+c) c(6+c)(-3+2 c)} \partial T \bar{D} T \\
& \left.+\frac{(-12+7 c)}{2 c}(D V \pm \bar{D} V)\right](w) \\
& +\frac{1}{(z-w)}\left[-\frac{(-15+c) c}{(3+c)(-12+5 c)} \alpha \partial^{2}(D W \mp \bar{D} W)+\frac{(-3+c)\left(9-3 c+c^{2}\right)}{(-1+c)(6+c)(-3+2 c)} \partial^{3} D T\right. \\
& -\frac{6}{c} T(D V \mp \bar{D} V)-\frac{54(6+c)}{c(3+c)(-12+5 c)} \alpha T T(D W \mp \bar{D} W) \\
& -\frac{27(-15+c)}{(-1+c) c(6+c)(-3+2 c)} T T T(D T \mp \bar{D} T)+\frac{36(-15+c)}{c(3+c)(-12+5 c)} \alpha T(D T \mp \bar{D} T) W \\
& +\frac{81}{(6+c)(-3+2 c)} T([\widetilde{D, \bar{D}}] T D T \mp \bar{D} T[\widetilde{D, \bar{D}}] T)-\frac{6}{c} W(D W \mp \bar{D} W) \\
& +\frac{6\left(54-6 c+c^{2}\right)}{c(3+c)(-12+5 c)} \alpha T(\partial D W \pm \partial \bar{D} W)-\frac{3}{(3+c)} \alpha(D T \mp \bar{D} T)[D, \widetilde{\bar{D}}] W \\
& +\frac{3(24+11 c)}{(3+c)(-12+5 c)} \alpha(D T \pm \bar{D} T) \partial W+\frac{12\left(72-27 c+4 c^{2}\right)}{c(3+c)(-12+5 c)} \alpha(\partial D T \pm \partial \bar{D} T) W \\
& -\frac{27 c}{(6+c)(-3+2 c)}(\partial D T \pm \partial \bar{D} T)[D \widetilde{D,}] T \pm \frac{(-3+c)^{2}\left(-9+2 c^{2}\right)}{2(-1+c) c(6+c)(-3+2 c)} \partial^{3} \bar{D} T \\
& \pm \frac{54\left(-9+2 c^{2}\right)}{(-1+c) c(6+c)(-3+2 c)} \partial \bar{D} T T T-\frac{9(-3+c)}{(-1+c)(-3+2 c)}(\partial D T \mp \partial \bar{D} T) \partial T \\
& \left. \pm \frac{9(-3+c)(3+c)(-9+2 c)}{2(-1+c) c(6+c)(-3+2 c)} \partial^{2} \bar{D} T T+\frac{3}{(3+c)} \alpha \widetilde{[D, \bar{D}}\right] T(D W \mp \bar{D} W) \\
& -\frac{3\left(18-18 c+7 c^{2}\right)}{(-1+c)(6+c)(-3+2 c)} \partial[\widetilde{D, \bar{D}}] T(D T \pm \bar{D} T) \\
& +\frac{9\left(36-81 c+17 c^{2}\right)}{(-1+c) c(6+c)(-3+2 c)} \partial T T D T+\frac{54(-9+2 c)}{(-1+c)(6+c)(-3+2 c)} \partial D T T T
\end{aligned}
$$




$$
\begin{aligned}
& -\frac{9\left(36-9 c+c^{2}\right)}{(-1+c)(6+c)(-3+2 c)} \partial^{2} D T T+\frac{3(-36+c)(-6+c)}{c(3+c)(-12+5 c)} \alpha(\partial T D W \pm \partial T \bar{D} W) \\
& -\frac{3(-6+c)\left(9-3 c+c^{2}\right)}{(-1+c) c(6+c)(-3+2 c)} \partial^{2} T(D T \mp \bar{D} T) \pm \frac{9\left(-18-27 c+17 c^{2}\right)}{(-1+c) c(6+c)(-3+2 c)} \partial T T \bar{D} T \\
& +2 \partial(D V \pm \bar{D} V)](w)+\cdots \\
& \longrightarrow \frac{1}{(z-w)^{4}} \frac{15}{2}[D T \pm \bar{D} T](w) \\
& +\frac{1}{(z-w)^{3}}\left[-\alpha(D W \mp \bar{D} W)+5 \partial(D T \pm \bar{D} T)-\frac{15}{c} T(D T \mp \bar{D} T)\right](w) \\
& +\frac{1}{(z-w)^{2}}\left[-\frac{3}{5} \alpha \partial(D W \mp \bar{D} W)+\frac{15}{8} \partial^{2}(D T \pm \bar{D} T)+\frac{9}{5 c} \alpha T(D W \pm \bar{D} W)\right. \\
& +\frac{315}{4 c^{2}} T T(D T \pm \bar{D} T)+\frac{69}{5 c} \alpha(D T \pm \bar{D} T) W-\frac{33}{8 c}[\widetilde{\bar{D}}] T(D T \pm \bar{D} T) \\
& \left.-\frac{45}{4 c}(\partial D T \mp \partial \bar{D} T) T-\frac{45}{8 c} \partial T(D T \mp \bar{D} T)+\frac{7}{2}(D V \pm \bar{D} V)\right](w) \\
& +\frac{1}{(z-w)}\left[-\frac{1}{5} \alpha \partial^{2}(D W \mp \bar{D} W)+\frac{1}{2} \partial^{3}(D T \pm \bar{D} T)\right. \\
& -\frac{6}{c} T(D V \mp \bar{D} V)-\frac{54}{5 c^{2}} \alpha T T(D W \mp \bar{D} W) \\
& -\frac{27}{2 c^{3}} T T T(D T \mp \bar{D} T)+\frac{36}{5 c^{2}} \alpha T(D T \mp \bar{D} T) W+\frac{81}{2 c^{2}} T[\widetilde{\bar{D}}] T(D T \mp \bar{D} T) \\
& -\frac{6}{c} W(D W \mp \bar{D} W)+\frac{6}{5 c} \alpha T(\partial D W \pm \partial \bar{D} W)-\frac{3}{c} \alpha(D T \mp \bar{D} T)[D, \widetilde{\bar{D}}] W \\
& +\frac{33}{5 c} \alpha(D T \pm \bar{D} T) \partial W+\frac{48}{5 c} \alpha(\partial D T \pm \partial \bar{D} T) W-\frac{27}{2 c}(\partial D T \pm \partial \bar{D} T)[D \bar{D}] T \\
& \frac{54}{c^{2}}(\partial D T \pm \partial \bar{D} T) T T-\frac{9}{2 c}(\partial D T \mp \partial \bar{D} T) \partial T+\frac{3}{c} \alpha[D, \bar{D}] T(D W \mp \bar{D} W) \\
& -\frac{21}{2 c} \partial[D \widetilde{\bar{D}}] T(D T \pm \bar{D} T)+\frac{153}{2 c^{2}} \partial T T(D T \pm \bar{D} T)-\frac{9}{2 c}\left(\partial^{2} D T \mp \partial^{2} \bar{D} T\right) T \\
& +\frac{3}{5 c} \alpha \partial T(D W \pm \bar{D} W)-\frac{3}{2 c} \partial^{2} T(D T \mp \bar{D} T) \\
& +2 \partial(D V \pm \bar{D} V)](w)+\cdots \\
& +2
\end{aligned}
$$

The relative coefficients $\frac{15}{2}, 5, \frac{15}{8}$, and $\frac{1}{2}$ in the spin- $\frac{3}{2}$ field and its descendant fields can be obtained from the coefficient formula in $[48,1]$. The relative coefficients $1, \frac{3}{5}$, and $\frac{1}{5}$ in the spin $-\frac{5}{2}$ field and its descendant fields can be obtained similarly.

Finally, one has the following operator product expansion between the spin-3 and the spin-3

$$
(-1) \frac{1}{2}[D, \bar{D}] W(z)(-1) \frac{1}{2}[D, \bar{D}] W(w)=\frac{1}{(z-w)^{6}} \frac{1}{2}(-12+5 c)
$$




$$
\begin{aligned}
& +\frac{1}{(z-w)^{4}}\left[\frac{3(-8+c)}{c} \alpha W-\frac{3(-12+5 c)}{2(-1+c)}[D \widetilde{\bar{D}}] T+\frac{9(-4+5 c)}{2(-1+c) c} T^{2}\right](w) \\
& +\frac{1}{(z-w)^{3}}\left[\frac{3(-8+c)}{2 c} \alpha \partial W-\frac{3(-12+5 c)}{4(-1+c)} \partial[\widetilde{D, \bar{D}}] T+\frac{9(-4+5 c)}{2(-1+c) c} \partial T T\right](w) \\
& +\frac{1}{(z-w)^{2}}\left[\frac{3\left(-12-19 c+3 c^{2}\right)}{4(3+c)(-12+5 c)} \alpha \partial^{2} W-\frac{9 c\left(30-11 c+2 c^{2}\right)}{8(-1+c)(6+c)(-3+2 c)} \partial^{2}[D, \bar{D}] T\right. \\
& -\frac{36}{c} T V-\frac{18(24+11 c)}{c(3+c)(-12+5 c)} \alpha T[D \widetilde{D}] W+\frac{72(6+c)(6+5 c)}{c^{2}(3+c)(-12+5 c)} \alpha T T W \\
& -\frac{18\left(-54-3 c+8 c^{2}\right)}{(-1+c) c(6+c)(-3+2 c)} T T[\widetilde{D, \widetilde{D}}] T \\
& +\frac{108(-3+c)(3+4 c)}{(-1+c) c^{2}(6+c)(-3+2 c)} T^{4}-\frac{12}{c} W^{2}+\frac{63(-6+c)}{(-1+c)(6+c)(-3+2 c)} \partial[\widetilde{D, \bar{D}}] T T \\
& +\frac{252(-6+c)}{(-1+c)(6+c)(-3+2 c)} T \bar{D} T D T+\frac{6(24+11 c)}{(3+c)(-12+5 c)} \alpha(\bar{D} T D W-D T \bar{D} W) \\
& +\frac{42(-6+c) c}{(-1+c)(6+c)(-3+2 c)}(\partial \bar{D} T D T+\partial D T \bar{D} T)-\frac{12(3+4 c)}{(3+c)(-12+5 c)} \alpha[\widetilde{[D, \bar{D}}] T W \\
& +\frac{3\left(18-27 c+16 c^{2}\right)}{2(-1+c)(6+c)(-3+2 c)}[\widetilde{D, \bar{D}}] T[\widetilde{D, \bar{D}}] T+\frac{54(48+c)}{(-1+c) c(6+c)(-3+2 c)} \partial T T T \\
& -\frac{9\left(-360+630 c-353 c^{2}+6 c^{3}\right)}{4(-1+c) c(6+c)(-3+2 c)} \partial^{2} T T-\frac{9\left(6-19 c+6 c^{2}\right)}{4(-1+c)(6+c)(-3+2 c)} \partial T \partial T \\
& \left.+\frac{6(3+4 c)}{(-1+c)(6+c)(-3+2 c)} \partial^{3} T-2[D, \bar{D}] V\right](w) \\
& +\frac{1}{(z-w)}\left[\frac{(-15+c) c}{2(3+c)(-12+5 c)} \alpha \partial^{3} W-\frac{c\left(30-11 c+2 c^{2}\right)}{4(-1+c)(6+c)(-3+2 c)} \partial^{3}[D \widetilde{D,}] T\right. \\
& -\frac{9(24+11 c)}{c(3+c)(-12+5 c)} \alpha \partial(T[D, \bar{D}] W)+\frac{3(24+11 c)}{(3+c)(-12+5 c)} \alpha(\bar{D} T \partial D W+\partial \bar{D} T D W) \\
& +\frac{126(-6+c)}{(-1+c)(6+c)(-3+2 c)}(\partial \bar{D} T T D T-\partial D T T \bar{D} T) \\
& +\frac{36(6+c)(6+5 c)}{c^{2}(3+c)(-12+5 c)} \alpha T T \partial W+\frac{72(6+c)(6+5 c)}{c^{2}(3+c)(-12+5 c)} \alpha \partial T T W-\frac{18}{c} \partial(T V)-\frac{12}{c} \partial W W \\
& +\frac{21(-6+c)(-3+c)}{(-1+c)(6+c)(-3+2 c)} \partial^{2} \bar{D} T D T-\frac{6(3+4 c)}{(3+c)(-12+5 c)} \alpha \partial([\widetilde{D, \bar{D}}] T W) \\
& +\frac{3\left(18-27 c+16 c^{2}\right)}{2(-1+c)(6+c)(-3+2 c)} \partial[\widetilde{D, \bar{D}}] T[\widetilde{D, \bar{D}}] T-\frac{3(24+11 c)}{(3+c)(-12+5 c)} \alpha \partial(D T \bar{D} W) \\
& -\frac{9\left(-54-3 c+8 c^{2}\right)}{(-1+c) c(6+c)(-3+2 c)} \partial[\widetilde{D, \bar{D}}] T T T+\frac{63(-6+c)}{2(-1+c)(6+c)(-3+2 c)} \partial[\widetilde{D, \bar{D}}] T \partial T \\
& +\frac{126(-6+c)}{(-1+c)(6+c)(-3+2 c)} \partial T \bar{D} T D T+\frac{21(-6+c)(-3+c)}{(-1+c)(6+c)(-3+2 c)} \partial^{2} D T \bar{D} T
\end{aligned}
$$




$$
\begin{aligned}
& -\frac{18\left(-54-3 c+8 c^{2}\right)}{(-1+c) c(6+c)(-3+2 c)} \partial T T[\widetilde{D, \bar{D}}] T-\frac{9\left(30-11 c+2 c^{2}\right)}{2(-1+c)(6+c)(-3+2 c)} \partial^{2} T \partial T \\
& +\frac{216(-3+c)(3+4 c)}{(-1+c) c^{2}(6+c)(-3+2 c)} \partial T T T T+\frac{27(48+c)}{(-1+c) c(6+c)(-3+2 c)} \partial T \partial T T \\
& \left.-\frac{3\left(-144+258 c-123 c^{2}+2 c^{3}\right)}{2(-1+c) c(6+c)(-3+2 c)} \partial^{3} T T+\frac{3(3+4 c)}{2(-1+c)(6+c)(-3+2 c)} \partial^{4} T-\partial[D, \bar{D}] V\right](w) \\
& +\cdots \\
& \longrightarrow \frac{1}{(z-w)^{6}} \frac{5 c}{2} \\
& +\frac{1}{(z-w)^{4}}\left[3 \alpha W-\frac{15}{2}[D \widetilde{\bar{D}}] T+\frac{45}{2 c} T^{2}\right](w) \\
& +\frac{1}{(z-w)^{3}} \frac{1}{2} \partial\left[3 \alpha W-\frac{15}{2}[D \widetilde{\bar{D}}] T+\frac{45}{2 c} T^{2}\right](w) \\
& +\frac{1}{(z-w)^{2}}\left[\frac{9}{20} \alpha \partial^{2} W-\frac{9}{8} \partial^{2}[D \widetilde{\bar{D}}] T-\frac{36}{c} T V+\frac{72}{c^{2}} \alpha T T W-\frac{72}{c^{2}} T T[D, \widetilde{\bar{D}}] T\right. \\
& +\frac{216}{c^{3}} T^{4}-\frac{12}{c} W^{2}+\frac{126}{c^{2}} T \bar{D} T D T+\frac{66}{5 c} \alpha(\bar{D} T D W-D T \bar{D} W) \\
& +\frac{21}{c}(\partial \bar{D} T D T+\partial D T \bar{D} T)-\frac{48}{5 c} \alpha[\widetilde{D, \bar{D}}] T W+\frac{12}{c}[\widetilde{D, \bar{D}}] T[\widetilde{D, \bar{D}}] T \\
& \left.-\frac{27}{8 c} \partial^{2} T^{2}-2[D, \bar{D}] V\right](w) \\
& +\frac{1}{(z-w)}\left[\frac{1}{10} \alpha \partial^{3} W-\frac{1}{4} \partial^{3}[D, \widetilde{\bar{D}}] T+\frac{33}{5 c} \alpha \partial(\bar{D} T D W-D T \bar{D} W)\right. \\
& +\frac{36}{c^{2}} \alpha \partial(T T W)-\frac{18}{c} \partial(T V)-\frac{6}{c} \partial(W W) \\
& +\frac{21}{2 c} \partial(\bar{D} T D T+D T \bar{D} T)-\frac{24}{5 c} \alpha \partial([\widetilde{\overline{,} \bar{D}}] T W)+\frac{6}{c} \partial([\widetilde{D, \bar{D}}] T[\widetilde{D, \bar{D}}] T) \\
& -\frac{36}{c^{2}} \partial(T T[\widetilde{D, \bar{D}}] T)+\frac{63}{c^{2}} \partial(T \bar{D} T D T)-\frac{3}{4 c} \partial^{3} T^{2} \\
& \left.+\frac{108}{c^{3}} \partial T^{4}-\partial[D, \bar{D}] V\right](w)+\cdots
\end{aligned}
$$

Due to the presence of $T^{2}(w)$ in the classical limit, its descendant fields, $\partial T^{2}(w), \partial^{2} T^{2}(w)$, and $\partial^{3} T^{2}(w)$ appear and the field $T \bar{D} T D T(w)$ occurs. Also the nonlinear term $T^{4}(w)$ and its descendant field $\partial T^{4}(w)$ appear in the right hand side. The relative coefficients of various spin-4 fields to its descendants, 1 and $\frac{1}{2}$, can be obtained from the conformal symmetry. Also note that the relative coefficients, $1, \frac{1}{2}, \frac{3}{20}$, and $\frac{1}{30}$ in the nonlinear $T^{2}$ term and its descendant fields are the same as the ones in linear spin-2 terms [16]. All of these do not appear in the bulk theory. Other remaining nonlinear terms appear in [13]. 
We also present the other operator product expansions

$$
\begin{aligned}
& (D W \pm \bar{D} W)(z) W(w)=-\frac{1}{(z-w)^{3}} 3[D T \mp \bar{D} T](w) \\
& +\frac{1}{(z-w)^{2}}\left[\alpha(D W \pm \bar{D} W)-\frac{(-3+2 c)}{(-1+c)} \partial(D T \mp \bar{D} T)-\frac{3}{(-1+c)} T(D T \pm \bar{D} T)\right](w) \\
& +\frac{1}{(z-w)}\left[-(D V \mp \bar{D} V)+\frac{3(-6+c)(-1+c)}{(3+c)(-12+5 c)} \alpha \partial(D W \pm \bar{D} W)\right. \\
& \pm \frac{3\left(27-18 c+3 c^{2}+2 c^{3}\right)}{4(-1+c)(6+c)(-3+2 c)} \partial^{2} \bar{D} T+\frac{6(-15+c)}{(3+c)(-12+5 c)} \alpha T(D W \mp \bar{D} W) \\
& -\frac{9(-15+c)}{2(-1+c)(6+c)(-3+2 c)} T T(D T \mp \bar{D} T)-\frac{54(-1+c)}{(3+c)(-12+5 c)} \alpha(D T \mp \bar{D} T) W \\
& +\frac{27 c}{2(6+c)(-3+2 c)}([\widetilde{\bar{D}}] T D T \mp \bar{D} T[\widetilde{D, \bar{D}}] T)-\frac{3 c\left(9-3 c+c^{2}\right)}{2(-1+c)(6+c)(-3+2 c)} \partial^{2} D T \\
& -\frac{3\left(-18+24 c+c^{2}\right)}{(-1+c)(6+c)(-3+2 c)} \partial D T T \mp \frac{3\left(9-3 c+c^{2}\right)}{(-1+c)(6+c)(-3+2 c)} \partial \bar{D} T T \\
& \left.-\frac{9(12+c)}{2(6+c)(-3+2 c)} \partial T D T \mp \frac{9(-6+c)}{2(6+c)(-3+2 c)} \partial T \bar{D} T\right](w)+\cdots \\
& \longrightarrow-\frac{1}{(z-w)^{3}} 3[D T \mp \bar{D} T](w) \\
& +\frac{1}{(z-w)^{2}}\left[\alpha(D W \pm \bar{D} W)-2 \partial(D T \mp \bar{D} T)-\frac{3}{c} T(D T \pm \bar{D} T)\right](w) \\
& +\frac{1}{(z-w)}\left[-(D V \mp \bar{D} V)+\frac{3}{5} \alpha \partial(D W \pm \bar{D} W) \pm \frac{3}{4} \partial^{2} \bar{D} T+\frac{6}{5 c} \alpha T(D W \mp \bar{D} W)\right. \\
& -\frac{9}{4 c^{2}} T T(D T \mp \bar{D} T)-\frac{54}{5 c} \alpha(D T \mp \bar{D} T) W+\frac{27}{4 c}([\overline{D, \bar{D}}] T D T \mp \bar{D} T[\widetilde{D, \bar{D}}] T) \\
& \left.-\frac{3}{2 c}(\partial D T \pm \partial \bar{D} T) T-\frac{9}{4 c} \partial T(D T \pm \bar{D} T)-\frac{3}{4} \partial^{2} D T\right](w)+\cdots,
\end{aligned}
$$

and

$$
\begin{aligned}
& (-1) \frac{1}{2}[D, \widetilde{\bar{D}}] W(z) W(w)=\frac{1}{(z-w)^{4}} 3 T(w)+\frac{1}{(z-w)^{3}} 3 \partial T(w) \\
& +\frac{1}{(z-w)^{2}}\left[-\frac{3(-8+c)}{2(-12+5 c)} \alpha[D, \widetilde{\bar{D}}] W-\frac{9 c(-12+5 c)}{4(-1+c)(6+c)(-3+2 c)} \partial[D \widetilde{\bar{D}}] T\right. \\
& +\frac{12}{c} \alpha T W-\frac{3\left(36-9 c+8 c^{2}\right)}{2(-1+c)(6+c)(-3+2 c)} T[\widetilde{\bar{D}}] T \\
& -\frac{27(-12+5 c)}{2(-1+c) c(6+c)(-3+2 c)} T^{3}-\frac{9 c(-12+5 c)}{(-1+c)(6+c)(-3+2 c)} \bar{D} T D T \\
& \left.-\frac{3\left(18-15 c+2 c^{2}+2 c^{3}\right)}{2(-1+c)(6+c)(-3+2 c)} \partial^{2} T+\frac{27(-12+5 c)}{2(-1+c)(6+c)(-3+2 c)} \partial T T+3 V\right](w)
\end{aligned}
$$




$$
\begin{aligned}
& +\frac{1}{(z-w)}\left[-\frac{(-15+c) c}{2(3+c)(-12+5 c)} \alpha \partial[D, \widetilde{\bar{D}}] W+\frac{6}{(3+c)} \alpha T \partial W\right. \\
& +\frac{6}{(3+c)} \alpha(\bar{D} T D W+D T \bar{D} W)-\frac{6 c(-12+5 c)}{(-1+c)(6+c)(-3+2 c)}(\partial \bar{D} T D T-\partial D T \bar{D} T) \\
& -\frac{6\left(9-3 c+c^{2}\right)}{(-1+c)(6+c)(-3+2 c)} \partial[D, \overline{\bar{D}}] T T+\frac{12}{(3+c)} \alpha \partial T W \\
& +\frac{3 c(3+4 c)}{(-1+c)(6+c)(-3+2 c)} \partial T[D, \overline{\bar{D}}] T-\frac{27(-12+5 c)}{(-1+c) c(6+c)(-3+2 c)} \partial T T T \\
& \left.+\frac{\left(18-15 c+2 c^{2}+2 c^{3}\right)}{2(-1+c)(6+c)(-3+2 c)} \partial^{3} T+2 \partial V\right](w)+\cdots \\
& -\frac{1}{(z-w)^{4}} 3 T(w)+\frac{1}{(z-w)^{3}} 3 \partial T(w) \\
& +\frac{1}{(z-w)^{2}}\left[-\frac{3}{10} \alpha[D, \widetilde{\bar{D}}] W+\frac{12}{c} \alpha T W-\frac{6}{c} T[\widetilde{\bar{D}}] T-\frac{45}{2 c} \bar{D} T D T-\frac{3}{2} \partial^{2} T+3 V\right](w) \\
& +\frac{1}{(z-w)}\left[-\frac{1}{10} \alpha \partial[D \widetilde{\bar{D}}] W+\frac{6}{c} \alpha T \partial W+\frac{6}{c} \alpha(\bar{D} T D W+D T \bar{D} W)\right. \\
& -\frac{15}{c}(\partial \bar{D} T D T-\partial D T \bar{D} T)-\frac{3}{c} \partial[D \widetilde{\bar{D}}] T T-\frac{12}{c} \alpha \partial T W+\frac{6}{c} \partial T[D, \widetilde{\bar{D}}] T \\
& \left.-\frac{18}{c^{2}} \partial T T T+\frac{1}{2} \partial^{3} T+2 \partial V\right](w) \\
& +\cdots,
\end{aligned}
$$

where the former can be obtained from (4.3) while the latter can be obtained from (4.4).

\section{References}

[1] P. Bouwknegt and K. Schoutens, "W symmetry in conformal field theory," Phys. Rept. 223, 183 (1993) hep-th/9210010].

[2] A. B. Zamolodchikov, "Infinite Additional Symmetries in Two-Dimensional Conformal Quantum Field Theory," Theor. Math. Phys. 65, 1205 (1985) [Teor. Mat. Fiz. 65, 347 (1985)].

[3] V. A. Fateev and S. L. Lukyanov, "The Models of Two-Dimensional Conformal Quantum Field Theory with Z(n) Symmetry," Int. J. Mod. Phys. A 3, 507 (1988).

[4] S. L. Lukyanov and V. A. Fateev, "Conformally Invariant Models Of Two-dimensional Qft With Z(n) Symmetry," Sov. Phys. JETP 67, 447 (1988). 
[5] S. L. Lukyanov and V. A. Fateev, "Physics reviews: Additional symmetries and exactly soluble models in two-dimensional conformal field theory," Chur, Switzerland: Harwood (1990) 117 p. (Soviet Scientific Reviews A, Physics: 15.2)

[6] F. A. Bais, P. Bouwknegt, M. Surridge and K. Schoutens, "Extensions of the Virasoro Algebra Constructed from Kac-Moody Algebras Using Higher Order Casimir Invariants," Nucl. Phys. B 304, 348 (1988).

[7] F. A. Bais, P. Bouwknegt, M. Surridge and K. Schoutens, "Coset Construction for Extended Virasoro Algebras," Nucl. Phys. B 304, 371 (1988).

[8] M. R. Gaberdiel and R. Gopakumar, "An $A d S_{3}$ Dual for Minimal Model CFTs," Phys. Rev. D 83, 066007 (2011) arXiv:1011.2986 [hep-th]].

[9] M. R. Gaberdiel and R. Gopakumar, "Triality in Minimal Model Holography," JHEP 1207, 127 (2012) [arXiv:1205.2472 [hep-th]].

[10] M. R. Gaberdiel and R. Gopakumar, "Minimal Model Holography," arXiv:1207.6697 [hep-th].

[11] T. Creutzig, Y. Hikida and P. B. Ronne, "Higher spin $A d S_{3}$ supergravity and its dual CFT," JHEP 1202, 109 (2012) [arXiv:1111.2139 [hep-th]].

[12] C. Candu and M. R. Gaberdiel, "Supersymmetric holography on $A d S_{3}$," arXiv:1203.1939 [hep-th].

[13] K. Hanaki and C. Peng, "Symmetries of Holographic Super-Minimal Models," arXiv:1203.5768 [hep-th].

[14] Y. Kazama and H. Suzuki, "New N=2 Superconformal Field Theories and Superstring Compactification," Nucl. Phys. B 321, 232 (1989).

[15] Y. Kazama and H. Suzuki, "Characterization of N=2 Superconformal Models Generated by Coset Space Method," Phys. Lett. B 216, 112 (1989).

[16] C. Ahn, "The Large N 't Hooft Limit of Kazama-Suzuki Model," JHEP 1208, 047 (2012) arXiv:1206.0054 [hep-th]].

[17] S. Banerjee, S. Hellerman, J. Maltz and S. H. Shenker, "Light States in Chern-Simons Theory Coupled to Fundamental Matter," arXiv:1207.4195] [hep-th]. 
[18] R. Gopakumar, A. Hashimoto, I. R. Klebanov, S. Sachdev and K. Schoutens, "Strange Metals in One Spatial Dimension," Phys. Rev. D 86, 066003 (2012) arXiv:1206.4719 [hep-th]].

[19] M. A. Vasiliev, "Holography, Unfolding and Higher-Spin Theory," arXiv:1203.5554 [hepth].

[20] M. R. Gaberdiel, T. Hartman and K. Jin, "Higher Spin Black Holes from CFT," JHEP 1204, 103 (2012) [arXiv:1203.0015 [hep-th]].

[21] M. Henneaux, G. Lucena Gomez, J. Park and S. -J. Rey, "Super- W(infinity) Asymptotic Symmetry of Higher-Spin $A d S_{3}$ Supergravity," JHEP 1206, 037 (2012) arXiv:1203.5152 [hep-th]].

[22] C. Ahn, "The Primary Spin-4 Casimir Operators in the Holographic SO(N) Coset Minimal Models," JHEP 1205, 040 (2012) [arXiv:1202.0074 [hep-th]].

[23] C. -M. Chang and X. Yin, "Correlators in $W_{N}$ Minimal Model Revisited," JHEP 1210, 050 (2012) arXiv:1112.5459 [hep-th]].

[24] M. Ammon, P. Kraus and E. Perlmutter, "Scalar fields and three-point functions in D=3 higher spin gravity," JHEP 1207, 113 (2012) [arXiv:1111.3926 [hep-th]].

[25] A. Castro, R. Gopakumar, M. Gutperle and J. Raeymaekers, "Conical Defects in Higher Spin Theories," JHEP 1202, 096 (2012) [arXiv:1111.3381 [hep-th]].

[26] C. Ahn, "The Coset Spin-4 Casimir Operator and Its Three-Point Functions with Scalars," JHEP 1202, 027 (2012) [arXiv:1111.0091 [hep-th]].

[27] K. Papadodimas and S. Raju, "Correlation Functions in Holographic Minimal Models," Nucl. Phys. B 856, 607 (2012) arXiv:1108.3077 [hep-th]].

[28] A. Campoleoni, S. Fredenhagen and S. Pfenninger, "Asymptotic W-symmetries in threedimensional higher-spin gauge theories," JHEP 1109, 113 (2011) arXiv:1107.0290 [hepth]].

[29] M. R. Gaberdiel and C. Vollenweider, "Minimal Model Holography for SO(2N)," JHEP 1108, 104 (2011) [arXiv:1106.2634 [hep-th]].

[30] C. -M. Chang and X. Yin, "Higher Spin Gravity with Matter in $A d S_{3}$ and Its CFT Dual," JHEP 1210, 024 (2012) [arXiv:1106.2580 [hep-th]]. 
[31] M. R. Gaberdiel, R. Gopakumar, T. Hartman and S. Raju, "Partition Functions of Holographic Minimal Models," JHEP 1108, 077 (2011) [arXiv:1106.1897 [hep-th]].

[32] C. Ahn, "The Large N 't Hooft Limit of Coset Minimal Models," JHEP 1110, 125 (2011) arXiv:1106.0351 [hep-th]].

[33] C. Candu and M. R. Gaberdiel, "Duality in $\mathrm{N}=2$ minimal model holography," arXiv:1207.6646 [hep-th].

[34] C. M. Hull and B. J. Spence, "N=2 Current Algebra And Coset Models," Phys. Lett. B 241, 357 (1990).

[35] L. J. Romans, "The N=2 super W(3) algebra," Nucl. Phys. B 369, 403 (1992).

[36] S. Odake, "Superconformal Algebras and Their Extensions," Soryushiron Kenkyu(Kyoto) 78 (1989) 201 (in Japanese).

[37] C. Ahn, "Explicit construction of $\mathrm{N}=2 \mathrm{~W}(3)$ current in the $\mathrm{N}=2$ coset $\mathrm{SU}(3)$ / SU(2) $\mathrm{x}$ U(1) model," Phys. Lett. B 348, 77 (1995) hep-th/9410170.

[38] R. Blumenhagen and A. Wisskirchen, "Extension of the $\mathrm{N}=2$ virasoro algebra by two primary fields of dimension 2 and 3," Phys. Lett. B 343, 168 (1995) [hep-th/9408082].

[39] A. Wisskirchen, "Construction of $\mathrm{N}=2$ superW algebras," BONN-IB-94-21.

[40] S. Krivonos and K. Thielemans, "A Mathematica package for computing N=2 superfield operator product expansions," Class. Quant. Grav. 13, 2899 (1996) [hep-th/9512029].

[41] K. Thielemans, "A Mathematica package for computing operator product expansions," Int. J. Mod. Phys. C 2, 787 (1991).

[42] P. Bowcock, "Quasi-primary Fields And Associativity Of Chiral Algebras," Nucl. Phys. B 356, 367 (1991).

[43] C. Ahn, "Free superfield realization of $\mathrm{N}=2$ quantum super W(3) algebra," Mod. Phys. Lett. A 9, 271 (1994) hep-th/9304038.

[44] P. Bowcock and G. M. T. Watts, "On the classification of quantum W algebras," Nucl. Phys. B 379, 63 (1992) hep-th/9111062.

[45] C. Ahn, S. Krivonos and A. S. Sorin, "The Full structure of quantum N=2 superW(3)**2 algebra," Mod. Phys. Lett. A 10, 1299 (1995) hep-th/9501100. 
[46] C. Ahn, E. Ivanov, S. Krivonos and A. S. Sorin, "Quantum N=2 superW(2)(3) algebra in superspace," Mod. Phys. Lett. A 11, 1705 (1996) [hep-th/9512214].

[47] E. Ivanov and S. Krivonos, "Superfield realizations of $\mathrm{N}=2$ superW(3)," Phys. Lett. B 291, 63 (1992) [Erratum-ibid. B 301, 454 (1993)] hep-th/9204023.

[48] H. Lu, C. N. Pope, L. J. Romans, X. Shen and X. J. Wang, "Polyakov construction of the $\mathrm{N}=2$ superW(3) algebra," Phys. Lett. B 264, 91 (1991).

[49] M. R. Gaberdiel and T. Hartman, "Symmetries of Holographic Minimal Models," JHEP 1105, 031 (2011) [arXiv:1101.2910 [hep-th]].

[50] K. Ito, "Free field realization of $\mathrm{N}=2$ superW(3) algebra," Phys. Lett. B 304, 271 (1993) hep-th/9302039].

[51] H. T. Ozer, "On the superfield realization of superCasimir WA(n) algebras," Int. J. Mod. Phys. A 17, 317 (2002) [hep-th/0102203].

[52] R. Blumenhagen, M. Flohr, A. Kliem, W. Nahm, A. Recknagel and R. Varnhagen, "W algebras with two and three generators," Nucl. Phys. B 361, 255 (1991).

[53] H. G. Kausch and G. M. T. Watts, "A Study of W algebras using Jacobi identities," Nucl. Phys. B 354, 740 (1991).

[54] K. Hornfeck, "The Minimal supersymmetric extension of WA(n-1)," Phys. Lett. B 275, 355 (1992).

[55] K. Hornfeck, "W algebras with set of primary fields of dimensions $(3,4,5)$ and $(3,4,5$, 6)," Nucl. Phys. B 407, 237 (1993) [hep-th/9212104]. 